\title{
Topological defects in the crystalline state of one-component plasmas of non-uniform density
}

\author{
A. Mughal and M. A. Moore ${ }^{1}$ \\ ${ }^{1}$ School of Physics and Astronomy, University of Manchester, Manchester M13 9PL, U.K.
} (Dated: May 6, 2019)

\begin{abstract}
We study the ground state properties of classical Coulomb charges interacting with a $1 / r$ potential moving on a plane but confined either by a circular hard wall boundary or by a harmonic potential. The charge density in the continuum limit is determined analytically and is non-uniform. Because of the non-uniform density there are both disclinations and dislocations present and their distribution across the system is calculated and shown to be in agreement with numerical studies of the ground state (or at least low-energy states) of $N$ charges, where values of $N$ up to 5000 have been studied. A consequence of these defects is that although the charges locally form into a triangular lattice structure, the lattice lines acquire a marked curvature. A study is made of conformal crystals to illuminate the origin of this curvature. The scaling of various terms which contribute to the overall energy of the system of charges viz, the continuum electrostatic energy, correlation energy, surface energy (and so on) as a function of the number of particles $N$ is determined. "Magic number" clusters are those at special values of $N$ whose energies take them below the energy estimated from the scaling forms and are identified with charge arrangements of high symmetry.

PACS numbers: 61.72.Bb, 61.72.Mm, 61.72.Lk
\end{abstract}

\section{INTRODUCTION}

Classical charges moving on a plane and repelling each other via a Coulomb $1 / r$ potential have a ground state which is a triangular lattice (for appropriately chosen periodic boundary conditions). This is a two-dimensional (2D) example of Wigner crystallization and is known to occur in diverse areas of physics, such as electrons trapped in surface states of liquid helium [1], colloidal suspensions and quantum dots. In this paper we examine situations when the charges do not have a uniform density across the system. This occurs for example when the charges are confined by a circular hard wall or when they are confined by a harmonic potential.

From a geometrical view point a perfect crystal lattice is a periodically repeating arrangement of identical structural cells, which fit together without gaps or overlap. The question we seek to answer, for a classical Wigner crystal of non-uniform density, is: how much of what we mean by "crystal lattice" still applies to the resulting structure? On the one hand, one expects the structure of the lattice to be locally triangular; so that each lattice site has six nearest neighbors, since this is the optimal energy arrangement for uniform density. On the other hand, due to the changing density not all the symmetries of the triangular space group, such as the translational and rotational invariances can continue to apply. We seek to understand how this conflict is resolved. We are particularly interested in knowing if the resulting structure can be understood within the framework of elasticity theory, and if so, what role is played by plastic deformations, such as dislocations and disclinations. In addition, using a continuum model, we shall attempt to quantify the scaling with $N$ of various phenomena associated with the structure of the cluster of charges, such as the energy, correlation energy (see below for its definition), surface energy and so on. This will in turn allow us to develop a link between symmetry and the energy of the lattice; we expect states with a high degree of symmetry to have a particularly low energy. Such states are known in the literature as "magic number" states.

An experimental realization of a system of $2 \mathrm{D}$ charges confined by a hard wall might be electrons trapped in surface states of liquid ${ }^{4} \mathrm{He}[1]$. The hard wall potential could be effected by a circular boundary made from an electrical insulator. A harmonic confining potential also produces a non-uniform density across the system and has been studied by Koulakov and Shklovskii, see [2] and [3]. Their study is relevant to the properties of quantum dots. It was found that the density of the charges was greatest at the center and diminished, upon approaching the edge (whereas with hard-wall confinement, the density rises from the center of the disc towards the wall because of the repulsion between the charges). Their simulations showed that although the charge density was not uniform, nevertheless the lattice was locally triangular. They showed that this is possible because the changing lattice density is accompanied by plastic deformations.

It is a consequence of topology that both the hardwall and the harmonic potential systems must contain an excess of 6 positive disclinations or pentagonal regions. Furthermore, the changing density introduces disclinations throughout the cluster. Disclinations also occur at the edges of the clusters to allow the lattice to adapt to the imposed circular structure. In addition to disclinations, the cluster includes dislocations (a tightly bound five-seven coordinated disclination pair). Unlike disclinations the total number of dislocations is not fixed by topology; dislocations are present to reduce the strain energy in the crystal which is induced by the circular edge and the disclinations. Dislocations are present in large numbers near the lattice edge, where they form a cloud 
around any disclinations and help reduce the large elastic stress induced by the latter. However, it was found by Koulakov and Shklovskii that disclinations and dislocations are to be found also in the lattice interior and act as a mechanism for reducing the strain energy there.

A number of other authors have carried out numerical simulations in situations where the classical 2D Wigner crystal has a non-uniform density. Bedanov and Peeters [4] considered particles interacting via the pure Coulomb potential and confined either by a hard wall or a parabolic potential. Simulations on the hard wall problem have also been carried out by Kong et al [5]; in addition to the Coulomb interaction they also consider the dipole and Yukawa potentials. Ying-Ju Lin and Lin I [6] have studied a number of systems with different interaction and confining potentials. Our work confirms and extends these earlier numerical studies, but also includes an account of our attempts to understand the numerical results.

The paper is organized as follows. In Sec. II, we present the systems we study and our numerical approach. In Sec. III we expand on the work by Koulakov and Shklovskii and derive the continuum approximation for the cluster of charges. We can then give an analytical calculation for the density of charges in the continuum limit, and for the density of dislocations and disclinations. We give a series expansion which we believe describes the various contributions to the ground state energy of the cluster. These results are compared to numerical experiments in Sec. IV. The discussion is in Sec. $\mathrm{V}$, especially of the striking lattice curvature effect visible in our studies of large clusters which is compared to that seen in conformal crystals.

\section{NUMERICAL APPROACH}

The energy of a cluster of $\mathrm{N}$ charges confined to a disk of radius $\mathrm{R}$, by a hard-wall potential is given by

$$
E^{H}=\sum_{i}^{N} V\left(\mathbf{r}_{i}\right)+\sum_{i<j}^{N} \frac{1}{\left|\mathbf{r}_{i}-\mathbf{r}_{j}\right|},
$$

where

$$
V\left(\mathbf{r}_{i}\right)= \begin{cases}0 & \text { for } r_{i}<R \\ \infty & \text { for } r_{i} \geq R\end{cases}
$$

The energy of a cluster of $\mathrm{N}$ charges in a parabolic confining potential is given by

$$
E^{P}=A \sum_{i}^{N} r_{i}{ }^{2}+\sum_{i<j}^{N} \frac{1}{\left|\mathbf{r}_{i}-\mathbf{r}_{j}\right|},
$$

where we set $A=1 / 2$.

Finding the global minimum for a function such as $E^{H}$ or $E^{P}$ is a very difficult task. The number of metastable states proliferate exponentially with $N$; consequentially the global minimum is obscured by a vast number of local minima with energies close to that of the global minimum. There exist a number of heuristic methods for such problems. Although there is no guarantee of finding the global minimum, it is possible to find states close to it.

We found that for the hard-wall system the standard Metropolis simulated annealing algorithm to be more effective than a conjugate gradient algorithm [7]. For a system with $N$ charges the simulated annealing algorithm was run with typically $N \times\left(5 \times 10^{6}\right)$ Monte Carlo steps. The temperature of the simulation was decreased linearly. The average displacement of the charges at each temperature step was chosen by an automatic process to give an acceptance probability of $0.5 \pm 0.01$. Promising states were reheated and annealed repeatedly to iron out as many defects as possible. Finally the results were put through a conjugate gradient algorithm to remove any residual strains.

For the harmonic (parabolic) potential case, we found the conjugate gradient algorithm to be as effective as simulated annealing. We used the former method for this system as it ran faster. Results were generated starting from an initial random configuration, which had a radial density profile matching the continuum limit density given below in Eq. (12).

\section{THE CONTINUUM LIMIT}

In the following we develop the continuum model of the two systems. For the case of parabolic confinement, many of the important results have already been derived by Koulakov and Shklovskii, see [2] and [3], so where appropriate we shall simply quote the relevant result. The bulk of the material in this section is concerned with the system with a hard-wall confining potential.

In the following, for the system with the hard-wall confining potential, we use a variational approach to derive the (non-uniform) charge density in the continuum limit. Next we demonstrate that as a consequence of the nonuniform density the system interior will contain topological defects, where the density of these defects depends on the rate of change of the density. Finally, for the system with a hard-wall confining potential, a series is developed which includes the contributions to the energy of the cluster which scale smoothly with system size.

\section{A. Charge Density}

For charges confined by a hard-wall potential, the energy expression in Eq. (11) can be approximated by the integrals over the disc $r \leq R$,

$$
E=\frac{1}{2} \int d^{2} r \int d^{2} r \frac{\rho^{H}(\mathbf{r}) \rho^{H}\left(\mathbf{r}^{\prime}\right)}{\left|\mathbf{r}-\mathbf{r}^{\prime}\right|} .
$$

The continuum approximation treats the density $\rho^{H}(\mathbf{r})$ as a smooth function rather than the sum of delta func- 
tions

$$
\rho^{H}(\mathbf{r})=\sum_{i=1}^{N} \delta\left(\mathbf{r}-\mathbf{r}_{i}\right),
$$

where $\mathbf{r}_{i}$ is the position of the $i^{t h}$ charge. One then minimizes the energy of the cluster, with respect to the smooth function $\rho^{H}(\mathbf{r})$, subject to the constraint that the number of particles

$$
N=\int d^{2} r \rho^{H}\left(\mathbf{r}^{\prime}\right),
$$

is constant. Introducing the Lagrange multiplier $\mu$ the constrained equation is

$$
E=\int \rho^{H}\left(\mathbf{r}^{\prime}\right)\left[\frac{1}{2} \int d^{2} r \frac{\rho^{H}(\mathbf{r})}{\left|\mathbf{r}-\mathbf{r}^{\prime}\right|}-\mu\right] d^{2} r^{\prime} .
$$

A variation in the energy is given by

$$
\delta E=E\left[\rho^{H}(\mathbf{r})+\delta \rho^{H}(\mathbf{r})\right]-E\left[\rho^{H}(\mathbf{r})\right],
$$

where $\delta \rho^{H}(\mathbf{r})$ represents a small change in the charge density. Keeping only terms up to first order, Eq. (6) gives

$$
\delta E=\int \delta \rho^{H}\left(\mathbf{r}^{\prime}\right)\left[\int d^{2} r \frac{\rho^{H}(\mathbf{r})}{\left|\mathbf{r}-\mathbf{r}^{\prime}\right|}-\mu\right] d^{2} r^{\prime} .
$$

To make the functional derivative stationary we require

$$
\int d^{2} r \frac{\rho^{H}(\mathbf{r})}{\left|\mathbf{r}-\mathbf{r}^{\prime}\right|}-\mu=0 .
$$

To solve this integral equation it is convenient to write the integral in Eq. (8) in terms of radial and angular variables:

$$
\begin{aligned}
\mu & =\int_{0}^{R} \rho^{H}(r) r d r \int_{0}^{2 \pi} \frac{d \theta}{\left(r^{2}+r^{\prime 2}-2 r r^{\prime} \cos \theta\right)^{\frac{1}{2}}} \\
& =\int_{0}^{R} d r \frac{4 r \rho^{H}(r)}{r+r^{\prime}} K\left(\frac{2 \sqrt{r r^{\prime}}}{r+r^{\prime}}\right),
\end{aligned}
$$

where $K(k)$ is an elliptical integral of the first kind. This is a Fredholm integral equation of the first kind which has the radially symmetric solution $[8]$

$$
\rho^{H}(r)=\frac{\mu}{\pi^{2}} \frac{1}{\sqrt{R^{2}-r^{2}}} .
$$

To determine the Lagrange multiplier we substitute Eq. (9) into Eq. (5), which gives $\mu=N \pi / 2 R$, and we finally have

$$
\rho^{H}(r)=\frac{N}{2 \pi R^{2}} \frac{1}{\sqrt{1-\left(\frac{r}{R}\right)^{2}}} .
$$

Incidentally, this result is the density profile obtained if a hemispherical shell of charge is projected onto a plane.
It is important to note the physical meaning of the Lagrange multiplier $\mu$; it is the electric potential at any point inside the disk when the charge is distributed according to Eq. (10). This can be seen by referring back to the original formulation of the problem as given by Eq. (8).

The number of charges within a distance $r$ from the center of the disc is given by integrating Eq. (10) over the region $r^{\prime} \leq r$ :

$$
N^{H}(r)=N\left(1-\sqrt{1-\left(\frac{r}{R}\right)^{2}}\right) .
$$

The charge density in the continuum limit for a cluster of charges in a parabolic confining potential is [3]

$$
\rho^{P}(r)=\rho_{o} \sqrt{1-\left(\frac{r}{R}\right)^{2}},
$$

where

$$
R=\left(\frac{3 \pi N}{8 A}\right)^{\frac{1}{3}} \text { and } \rho_{o}=\frac{4 A R}{\pi^{2}} .
$$

The total number of charges within a distance $r$ from the center of the disc is

$$
N^{P}(r)=N\left(1-\left(1-\left(\frac{r}{R}\right)^{2}\right)^{\frac{3}{2}}\right) .
$$

\section{B. Density of Defects}

Volterra dislocations are plastic imperfections characteristic of a deformed solid [9]. For a 2D lattice the only relevant Volterra dislocations are the edge dislocations and the wedge disclination; these we discuss in turn below.

A dislocation in a perfect lattice can be created by making a cut in the lattice, translating the cut edges with respect to each other and inserting/removing material. This process can also be viewed as the insertion/removal of a half plane of atoms, it is characterized by a discrete Burgers vector $\mathbf{B}$ which measures the amount by which the Burgers circuit around the dislocation fails to close 10].

A disclination can be created in a perfect lattice by making a cut, rotating the cut edges with respect to each other (this then defines a disclination axis) and inserting/removing a wedge of material. After the wedge is inserted/removed the whole construction is welded together and allowed to relax. The closure failure of a Burgers circuit around a disclination is given by

$$
\mathrm{B}=\boldsymbol{\Omega} \times \mathbf{r},
$$

where $\mathbf{r}$ is the distance from the disclination axis $\boldsymbol{\Omega}$, and $|\boldsymbol{\Omega}|$ is the wedge angle [11]. In a real lattice the wedge angle is quantized by the lattice symmetry, thus in a 
triangular lattice matter is inserted into the lattice if $|\boldsymbol{\Omega}|=+2 \pi / 6$ and removed if $|\boldsymbol{\Omega}|=-2 \pi / 6$, which corresponds to a heptagon or a pentagon in the crystal lattice respectively.

It is important to know the relationship between dislocations and disclinations. A dislocation is a tightly bound pair of disclinations of the opposite sign. A disclination can be decomposed into a series of dislocations, each of which have the same sign. For an illustration of both these points see [11].

Disclinations can be present in the ground state of a system for two reasons; either because they are demanded by topology (a consequence of Euler's theorem), or because the lattice density is non-uniform.

The first point has been covered in depth elsewhere [3], it will suffice to say that a Delaunay triangulation of a lattice will produce a unique planar graph. Euler's theorem states that for any such graph in flat space the following relationship holds between the number of vertices $\mathrm{v}$, edges e and faces $\mathrm{f}$

$$
v+f-e=1 \text {. }
$$

As a consequence of applying Euler's theorem to a triangular lattice we can assign a topological charge to each lattice site (or vertex), the sign and magnitude of the charge depends on by how much the coordination number (i.e. the number of nearest neighbors the site has) differs from 6 . For example a pentagon has a topological charge +1 while a square has a charge of +2 . Similarly a heptagon has a topological charge of -1 while an octagon has -2 . Obviously a hexagon is topologically neutral. The total topological charge for any cluster is always conserved and must always be equal to +6 .

We now show that a change in the density of the lattice leads to a closure failure of the Burgers circuit. This will allow us to calculate the Burgers vector density, from which the density of dislocations and disclinations can be determined.

Consider a lattice with smoothly varying density and let us suppose that the increase in density depends only on the radial distance r. Drawing a square of dimensions $\Delta r$ around such a region of lattice, see Fig. 1, we see that the number of lattice rows crossing the side $p q$ is given by

$$
L(r)=\frac{\Delta r}{a(r)},
$$

and the number crossing the side $s r$ is given by

$$
L(r+\Delta r)=\frac{\Delta r}{a(r+\Delta r)},
$$

where a(r), the lattice spacing, is a function of density and in a triangular lattice is given by

$$
a(r)=\sqrt{\frac{2}{\rho(r) \sqrt{3}}} .
$$

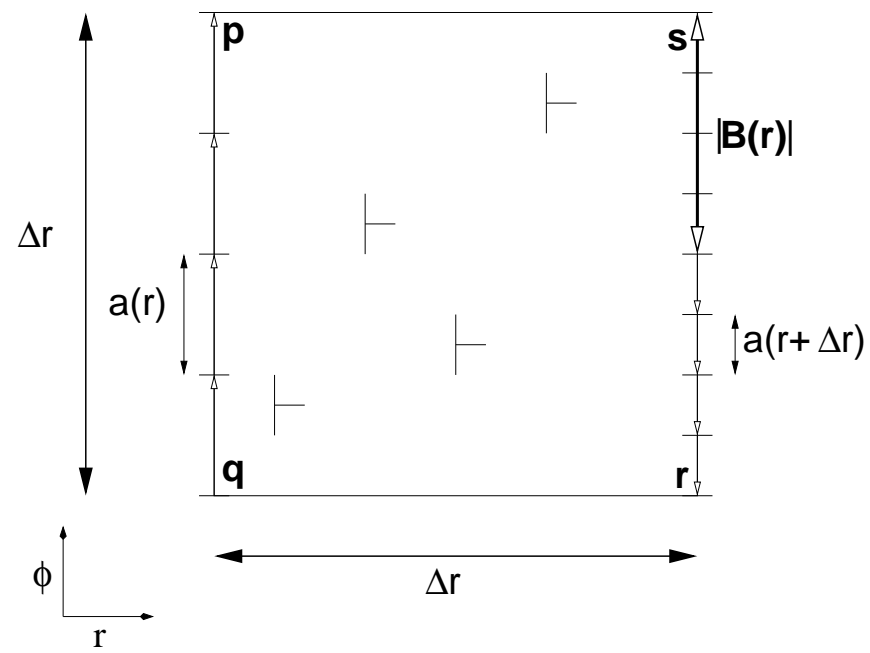

FIG. 1: Consider a region of crystal in which the lattice density is increasing in the $\mathrm{r}$ direction. It can be seen that by drawing a square Burgers circuit (of dimension $\Delta r$ ) around the region that there are more lattice lines crossing the side $s r$ than crossing the side $p q$ (while the number of lattice lines crossing the side $p s$ is equal to the number crossing the side $q r)$ The difference in the number of lattice lines leads to a closure failure of the Burgers circuit. This closure failure implies that the square contains an excess of dislocations of the same sign, the sum of the length of their individual Burgers vector is equal to the length of the total Burgers vector. Note that the presence of a dislocation is indicated by a $\mathrm{T}$ like symbol. The vertical bar of the $\mathrm{T}$ symbol indicates the side on which the extra half plane of atoms is inserted into the lattice, while the horizontal top bar indicates the point at which the extra half plane of atoms terminates. Thus the Burgers vector is parallel to the top bar of the $\mathrm{T}$.

In constructing a Burgers circuit we take the same number of steps in the horizontal and vertical directions, however due to the change in the lattice spacing there will be a closure failure on the side $s r$. Assuming the closure failure is due to an excess of dislocations of the same sign (which are the origin of the extra half planes), then the total Burgers vector, due to all the dislocations enclosed, is given by

$$
B_{\phi}(r)=\Delta r-L(r) a(r+\Delta r),
$$

where $\mathrm{L}(\mathrm{r})$ is the number of steps taken in the Burgers circuit on the side $p q$, (note that the direction of the Burgers vector is perpendicular to the direction in which the density is increasing). From Eq. (16) we have

$$
\begin{aligned}
B_{\phi}(r) & =\Delta r-L(r) a(r+d r) \\
& =a(r+\Delta r)\left[\frac{\Delta r}{a(r+\Delta r)}-L(r)\right] \\
& =\Delta r a(r+\Delta r)\left[a^{-1}(r+\Delta r)-a^{-1}(r)\right] .
\end{aligned}
$$

Taylor expansion to first order yields

$$
B_{\phi}(r)=\Delta r a(r)\left[\left[\Delta r \frac{d a^{-1}(r)}{d r}\right] ;\right.
$$


to get the Burgers vector density we divide through by the area of the square and take the limit $\Delta r \rightarrow 0$, giving

$$
\begin{aligned}
b_{\phi}(r)=\lim _{\Delta r \rightarrow 0} \frac{B_{\phi}(r)}{(\Delta r)^{2}} & =a(r) \frac{d a^{-1}(r)}{d r} \\
& =\frac{1}{2} \frac{d}{d r} \ln \rho(r) .
\end{aligned}
$$

(Generalizing Eq. (17), the Burgers vector density in a $2 \mathrm{D}$ plane is given by the vector field [3]

$$
\mathbf{b}(\mathbf{r})=a(\mathbf{r}) \hat{\mathbf{z}} \times \nabla a^{-1}(\mathbf{r}),
$$

where $\hat{\mathbf{z}}$ is the unit vector perpendicular to the surface.)

The density of the Burgers vector is then

$$
b_{\phi}(r)= \pm \frac{r}{2 R^{2}} \frac{1}{1-\left(\frac{r}{R}\right)^{2}},
$$

where $b_{\phi}(r)$ is positive upon substituting Eq. (10) into Eq. (17) and negative upon substituting Eq. (12) into Eq. (17). This then defines a Burgers vector density field present throughout the lattice. The total Burgers vector within a radius $\mathrm{r}$ can be found by integrating Eq. (19) over the area of the disk.

We have assumed that the changing lattice density is due to the insertion of extra half planes into the lattice. Thus to get the density of dislocations, we divide the Burgers vector density by the distance between crystalline rows, $h=a \sqrt{3} / 2$, which gives for the hard wall case

$$
\rho_{d}^{H}(r)=\frac{b_{\phi}(r)}{h}=\sqrt{\frac{N}{4 \pi \sqrt{3}}} \frac{r}{R^{3}} \frac{1}{\left(1-\frac{r^{2}}{R^{2}}\right)^{\frac{5}{4}}},
$$

and there is a similar result for the parabolic case [3]. Hence, the number of dislocations within a radius $\mathrm{r}$ can be found by integrating Eq. (20) over the area $r^{\prime}<r$.

In addition to dislocations we expect disclinations in the lattice. These are present if the Burgers vector density is rotational. The density of disclination charge, $\tilde{s}(r)$, is given by the $\hat{\mathbf{z}}$ component of the curl of $\mathbf{b}(\mathbf{r})$ which reduces in our situation to

$$
\tilde{s}(r)=\frac{1}{r} \frac{\partial}{\partial r}\left(r b_{\phi}\right)=\frac{1}{2} \nabla^{2} \ln \rho(r)=R(r),
$$

where $R(r)$ is the scalar curvature and is equal to the density of disclination charge. This quantity is also known in the theory of plasticity as the incompatibility [12]. This relationship can be generalized to include free disclinations:

$$
\tilde{s}(\mathbf{r})=s(\mathbf{r})-\epsilon_{i k} \nabla_{k} b_{i}(\mathbf{r})=R(\mathbf{r}) .
$$

In analogy to a dielectric the $s(\mathbf{r})$ term is the charge density of the free disclinations, induced say as a consequence of the topology of the space in which the lattice is embedded, and $-\epsilon_{i k} \nabla_{k} b_{i}(\mathbf{r})$ is the polarization contribution from dislocations [9]. It turns out that for the hard wall case the six disclinations induced by the disk topology are always at the edge of the system. For the harmonic potential they are close to the edge in small systems, but as $N$ increases, the six topologically induced disclinations migrate towards the interior. Thus for values of $r$ away from the hard wall, the density of disclination charge, for the hard-wall system, can be found by substituting Eq. (10) into Eq. (21),

$$
\tilde{s}(\mathbf{r})=\frac{1}{2} \nabla^{2} \ln \rho(r)=\frac{1}{R^{2}} \frac{1}{\left(1-\frac{r^{2}}{R^{2}}\right)^{2}} .
$$

Integrating Eq. (23) over the area $r^{\prime}<r$ gives the total disclination charge within a radius $\mathrm{r}$

$$
\begin{aligned}
\Sigma(r) & =2 \pi \int_{0}^{r} r^{\prime} \tilde{s}\left(r^{\prime}\right) d r^{\prime} \\
& =\left(\frac{r}{R}\right)^{2} \frac{\pi}{1-\left(\frac{r}{R}\right)^{2}} .
\end{aligned}
$$

If the lattice only contains disclinations with charge $\frac{\pi}{3}$, then the number of disclinations within a given radius is

$$
N_{d i s c}(r)=\frac{\Sigma(r)}{\pi / 3}=\left(\frac{r}{R}\right)^{2} \frac{3}{1-\left(\frac{r}{R}\right)^{2}},
$$

where for the hard wall confined system we expect the lattice interior to contain an excess of 7 coordinated disclinations. For the harmonically confined system the number of 5 coordinated disclinations induced by the changing density can be similarly calculated and equals $N_{d i s c}(r)$. The fact that both the density of the Burgers vector Eq. (19) and the disclination charge density due to the changing density are equal but opposite for the two systems suggests that they are "mirror images" of each other. It makes sense then to compare and contrast the properties of these two systems.

\section{Smooth part of the energy}

In the following we examine the various terms which contribute to the overall energy of the system of charges in a hard wall confining potential. A similar study has already been made for the system with parabolic confinement [2]. We believe that the energy will have the form, as $N$ becomes large, of a series in decreasing powers of $N^{\frac{1}{2}}$ :

$$
E_{\text {Smooth }}=\kappa_{1} \frac{N^{2}}{R}+\kappa_{2} \frac{N^{\frac{3}{2}}}{R}+\kappa_{3} \frac{N}{R}+\kappa_{4} \frac{N^{\frac{1}{2}}}{R}+\kappa_{5},
$$

The first coefficient $\kappa_{1}=\pi / 4$; this is calculated next. We have obtained from our numerical estimates of the ground state energy of systems with varying values of $N$ the following estimates of the other coefficients: $\kappa_{2}=$ $-1.562033, \kappa_{3}=0.975852, \kappa_{4}=-0.008196$ and $\kappa_{5}=$ -0.307608 . 
The first term is the 'electrostatic energy'. It can be calculated by approximating the density by its continuum limit form, to give

$$
E_{E S}=\frac{1}{2} \int \rho(r) d^{2} r \int \rho\left(r^{\prime}\right) d^{2} r^{\prime} \frac{1}{\left|\mathbf{r}-\mathbf{r}^{\prime}\right|}
$$

upon recognizing the second integral as the electrostatic potential $\mu$, see Eq. (8), this can be written as

$$
E_{E S}=\mu \frac{1}{2} \int \rho\left(r^{\prime}\right) d^{2} r^{\prime}=\frac{\pi}{4} \frac{N^{2}}{R} .
$$

The next largest term is the 'correlation energy' which is the first correction to the continuum limit approximation because the charges are discrete. Koulakov and Shklovskii [2] suggested that it could be estimated by using the local density approximation (LDA), which states that for a large enough cluster, locally the density can be assumed to be constant, so the correlation energy of a lattice with non-uniform density should be the same as the first correction to the electrostatic energy of an infinite system of uniform density:

$$
\begin{aligned}
E_{\text {Corr }} & =-\frac{1}{2} \int_{0}^{R} 2 \pi r d r \rho(r) \beta \sqrt{\rho(r)} \\
& =-\beta \sqrt{\frac{1}{2 \pi}} \frac{N^{\frac{3}{2}}}{R},
\end{aligned}
$$

where the value of $\beta$ depends on the geometric properties of the lattice. For a triangular lattice $\beta=\beta_{\triangle}=3.921034$ and for a square lattice $\beta=\beta_{\square}=3.898598$ [13]. Visual inspection of the ground states of the system (see section IV.A.5) suggest that locally the lattice is triangular, thus in calculating the correlation energy we expect that $\beta=$ $\beta_{\triangle}$. If this expectation were correct it would predict that $\kappa_{2}=-1.5642939$, which is quite close to our numerical estimate -1.562033 . We discuss in the following sections some possible explanations for this small discrepancy and also how the remaining $\kappa$ coefficients were obtained.

\section{NUMERICAL RESULTS}

In this Section we present the results of our numerical simulations and compare them with the continuum model developed in Section III.

Simulated annealing experiments were carried out for the system with a hard wall boundary, as described in Section II, for systems ranging in size from $N=2$ to $N=100$. Our results either agreed with or gave a slightly lower energy than those published by Kong et al [5]. Simulated annealing experiments were also carried out for larger systems, $N=150,200,250,500,1000$, 2000 and 5000. To ensure good results a range of annealing schedules were tried. Upon finding the optimal schedule, minimization was repeated as many times as possible, thus not only improving the chances of finding

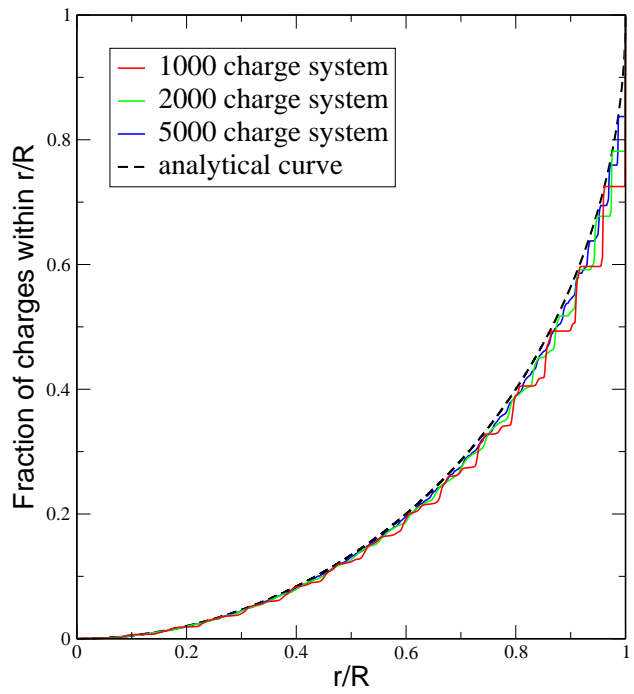

FIG. 2: Histograms of the fraction of the total charge of the system within a given radius $r$. Results for clusters containing 1000, 2000, and 5000 charges are colored red, green and blue respectively. The continuum limit result is given by the black dashed line. Note that the step-like behavior towards the edge indicates that the charge in this region is concentrated into a series of concentric shells; the charge arrangements in this area are very different from those in the cluster interior.

a good result but also generating a collection of states which could be used for further analysis.

For the system with parabolic confinement we restrict our efforts to large system i.e. $N=1000,2000$ and 5000. Numerical experiments have already been carried out for small systems by Koulakov and Shklovskii, see [2] and [3]. Starting with a random initial configuration of charges, a conjugate gradient algorithm was used to minimize the energy of the system. In each case this process was repeated 1000 times and the cluster with the lowest energy was identified.

For each system the best result was triangulated; this was done by projecting the charges onto a paraboloid and using the Delaunay triangulation package Qhull [14]. The results were then displayed using the graphics package Geomview [15]. An additional routine was used to highlight defects in the clusters, points with five nearest neighbors were colored red, while those with seven and eight nearest neighbors were colored green and blue respectively.

\section{A. Hard Wall System}

\section{Distribution of Charges}

In the continuum limit the charge is distributed according to Eq. (11); this quantity can be compared with its actual value in a finite sized cluster which we call 


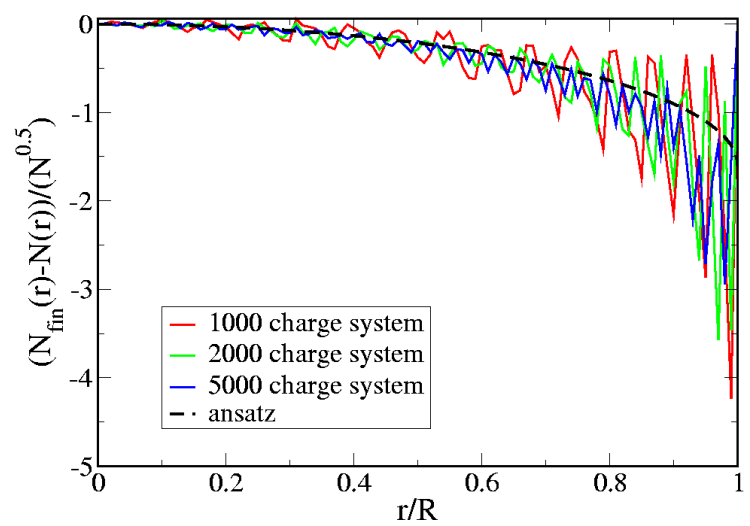

FIG. 3: Plot of $\Delta N$ for the three largest systems, scaled by $\sqrt{N}$ versus distance $r$ from the center. $\Delta N$ is the difference between the number of charges within a given radius for finite systems $N_{\text {fin }}(r)$ and the continuum result $N(r)$ (which is given by Eq. (11)). The black dotted line is an attempt to fit the data by replacing $N$ by $N-C N^{\frac{1}{2}}$ in Eq. (11) with $C \approx 1.6$.

$N_{f i n}(r)$, where for a given radius $\mathrm{r}, N_{\text {fin }}(r)$ is the number of charges enclosed within that radius. We choose to compare the integrated quantity as opposed to the charge density itself as this yields a less noisy result. Fig. 2 gives the fraction of the total charge enclosed as a function of radius, i.e. $N_{\text {fin }}(r) / N$, for the three largest systems simulated (in each case the result with the lowest energy is used). Also shown for comparison is the fraction of charge enclosed in the continuum limit, i.e. $N(r) / N$. By scaling the charge enclosed in this manner, different sized systems can be easily compared. The curves in Fig. 2 suggest that with increasing system size the charge distribution approaches the continuum result.

However, there is a systematic difference between the charge distribution in the continuum limit and that for finite sized systems, which is not just a local effect but varies on the scale of the radius $R$ of the system. Its presence is revealed on plotting $\Delta N(r)=N_{\text {fin }}(r)-N(r)$, for the three largest systems. There is a correction to the continuum expression for $N(r)$, which is of order $\sqrt{N}$. (The continuum expression for $N(r)$ is of order $N) . \Delta N(r) / \sqrt{N}$ is plotted in Fig. 3 for the three largest system sizes which we studied. It shows that there is a deficiency of charge in the system interior. Furthermore $\Delta N$ falls to zero only at the edge of the system, which means that the missing charge is to be found here in the form of extra charges, $N_{s}$ in number, at the surface. The leading term for the density is the result in the continuum limit and is of order $N / R^{2}$ as in Eq. (10); Fig. (3) shows that there are corrections to it of order $N^{\frac{1}{2}} / R^{2}$. We have been unable to obtain any analytic understanding of this correction term, but we have noticed that it can be quite well approximated by replacing $N$ in Eq. (10) by $N-N_{s}$ where $N_{s}=C N^{\frac{1}{2}}$ and $C \approx 1.6$. We shall estimate the number of charges in the outer shell of

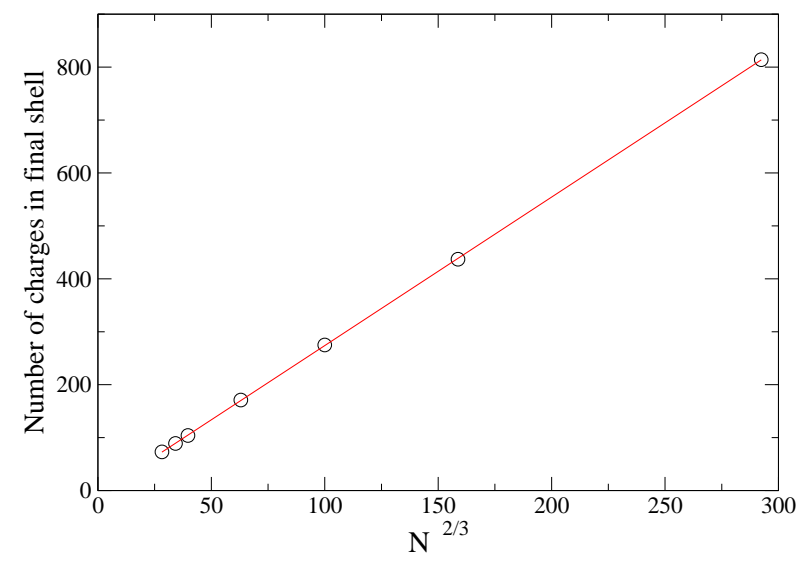

FIG. 4: A plot of $N_{\text {fin }}(R)$ against $N^{2 / 3}$ for systems in the range $\mathrm{N}=150$ to 5000 . To find $N_{\text {fin }}(R)$ we simply counted the number of charges in the outermost shell in each system.

the system i.e. on the hard wall, and show that in fact the "excess" surface charge $N_{s}$, (which is of order $N^{\frac{1}{2}}$ ) is only a small contribution to it at large $N$ as the number of charges in the outer shell increases as $N^{\frac{2}{3}}$.

The number of charges we would expect to find at the edge of the system can be estimated by the following argument: let the lattice spacing at the edge of the crystal be given by $a_{e}$, then the number of charges contained within a disk centered on the origin of radius $R-a_{e}$ is

$$
N\left(R-a_{e}\right)=N\left(1-\sqrt{1-(1-X)^{2}}\right),
$$

where $X=a_{e} / R$. Expanding the $(1-X)^{2}$ term to first order gives

$$
N\left(R-a_{e}\right) \approx N(1-\sqrt{2 X}) .
$$

Therefore the number of charges within a distance $a_{e}$ from the edge is

$$
N_{e}=N-N\left(R-a_{e}\right) \approx N \sqrt{\frac{2 a_{e}}{R}} .
$$

Imposing the condition that $N_{e}$ must be equal to the number of charges on the perimeter $N_{p}=2 \pi R / a_{e}$, yields the ratio

$$
\frac{R}{a_{e}} \approx\left(\frac{N}{\pi \sqrt{2}}\right)^{\frac{2}{3}},
$$

hence the number of charges on the edge, in the continuum limit, scales with $N^{\frac{2}{3}}$. To compare with the results of our numerical simulations we therefore plotted $N_{\text {fin }}(R)$, the total number of charges in the outermost 


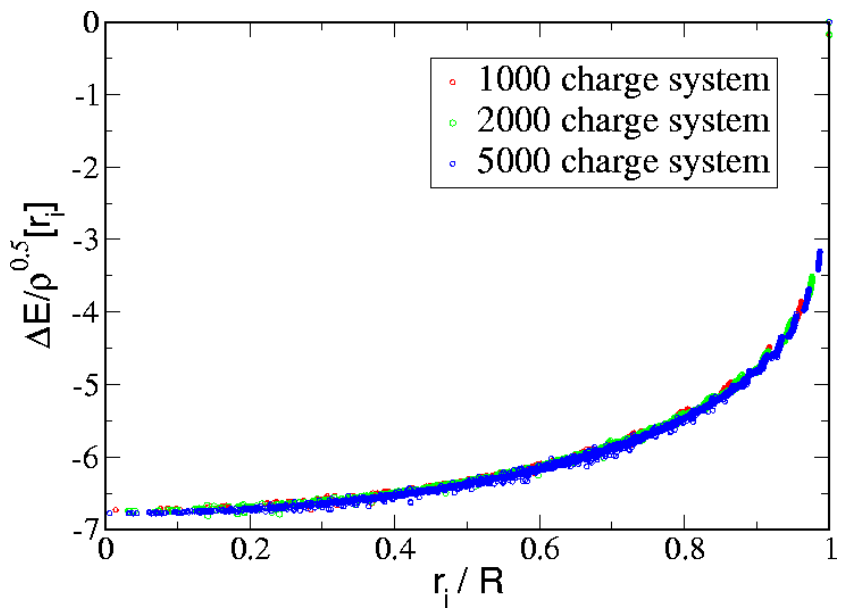

FIG. 5: Test of the LDA approximation. If the approximation were perfect $\Delta E\left(r_{i}\right) / \sqrt{\rho\left(r_{i}\right)}$ would be independent of $r_{i} / R$ and equal to the numerical value -3.921034 .

shell of each cluster, against $N^{2 / 3}$. As shown in Fig. 4 there is good agreement between these estimates and the results of our numerical simulations.

\section{The Local Density Approximation}

In Section III.C we calculated the correlation energy of the lattice using the local density approximation (LDA) which is based on the idea that locally the lattice appears to be triangular. Physically this seems to be a very reasonable approximation, but we observed that the numerical data was not in perfect agreement with this approximation. In this Section we shall investigate the matter further.

The correlation energy of a given charge will be defined as the difference in energy between the interaction of a charge with all the other charges in the system after the subtraction of the interaction of the charge with the continuum approximation for the other charges [13]:

$$
\begin{aligned}
\Delta E\left(r_{i}\right) & =\left[\sum_{j \neq i}^{N} \frac{1}{\left|\mathbf{r}_{i}-\mathbf{r}_{j}\right|}-\int \frac{\rho^{H}(r) d^{2} r}{\left|\mathbf{r}_{i}-\mathbf{r}\right|}\right] \\
& =\left[\sum_{j \neq i}^{N} \frac{1}{\left|\mathbf{r}_{i}-\mathbf{r}_{j}\right|}-\frac{\pi N}{2 R}\right]
\end{aligned}
$$

In the LDA, this quantity would be expected to equal $-\beta_{\triangle} \sqrt{\rho\left(r_{i}\right)}$. In Fig. (5) we have plotted $\Delta E\left(r_{i}\right) / \sqrt{\rho\left(r_{i}\right)}$ against $r_{i} / R$ to test this expectation. The agreement is non-existent.

The origin of this discrepancy is not hard to find. It arises because $\Delta E\left(r_{i}\right)$ is a quantity of size $N^{\frac{1}{2}} / R . \sqrt{\rho\left(r_{i}\right)}$ is of this magnitude, but so are also the contribution of the deviations of the density from $\rho^{H}(r)$ which are of order $N^{\frac{1}{2}} / R^{2}$. Alas, we do not have a calculation of these

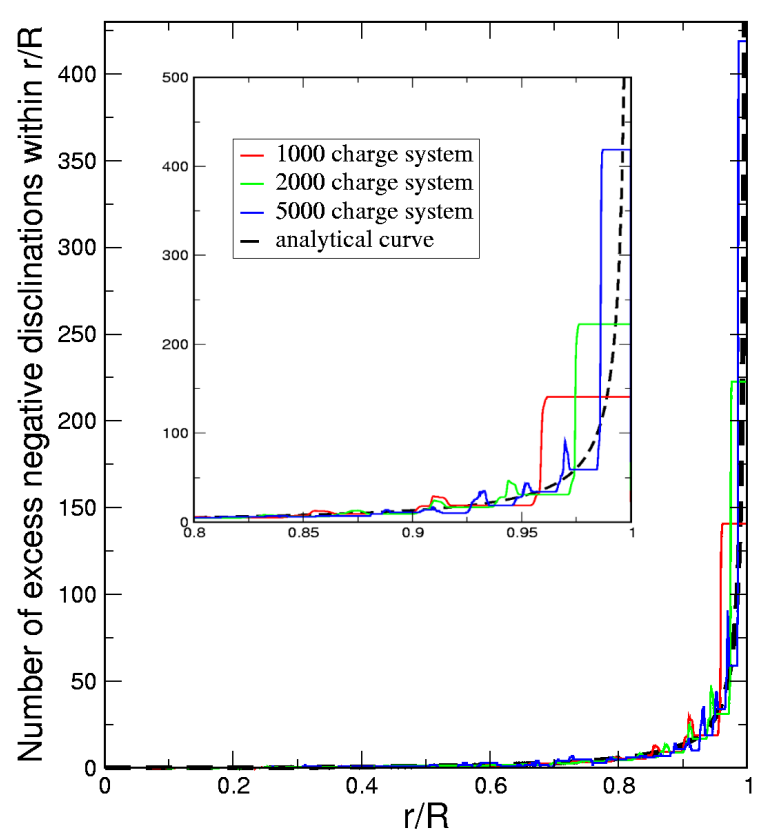

FIG. 6: Histogram giving the total number of disclinations within a given radius. Clusters containing 1000, 2000, and 5000 charges are colored red, green and blue respectively. The analytical curve is given by the black dashed line. The inset is a magnification giving the last $20 \%$ of the graph showing there is good agreement right up to the edge.

deviations. However, we have found that our attempts to model them by changing $N$ in the expression for $\rho^{H}(r)$ to $N-C \sqrt{(} N)$ and allowing for the compensating surface charge needed for charge neutrality does at least reduce the discrepancy in Fig. (5). So it could be that with proper allowance for these corrections to the density, the LDA might still be valid.

\section{Distribution of Disclinations}

The number of excess disclinations located within a given radius is predicted by Eq. (26). To compare this with our simulations we use the following method: for a given radius, we count the number of positive and negative disclinations enclosed, where we expect from Eq. (26) that in the interior of the lattice the number of negative disclinations will always be greater than the number of positive disclinations. We adopt the convention that a 7 coordinated point counts as one negative disclination, an 8 coordinated point as two negative disclinations and a 5 coordinated point counts as one positive disclination. In Fig. 6 we plot the number of excess negative disclinations within a given radius and compare with Eq. (26). It is evident that there is convergence with increasing system size. For every negative disclination in the lattice interior there is a compensating positive disclination on the lattice edge. In addition, the lattice edge also 


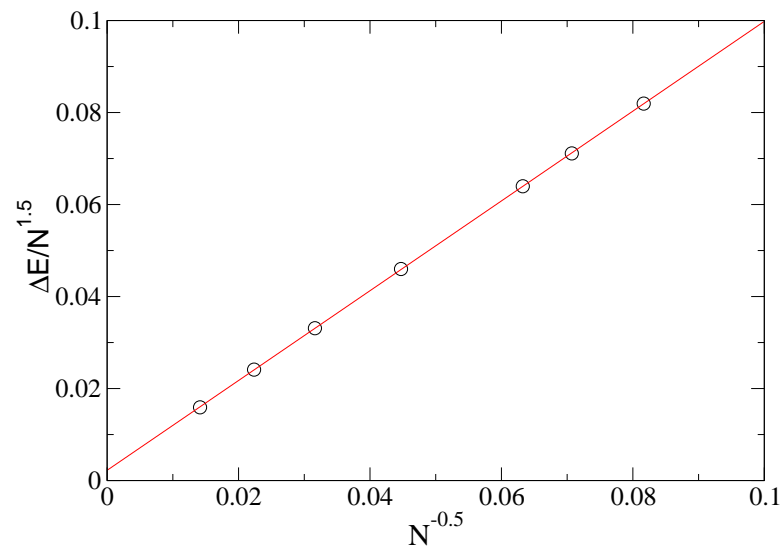

FIG. 7: $\frac{\Delta E}{N^{\frac{3}{2}}}$ against $N^{-} \frac{1}{2}$ plotted for $\mathrm{N}=150$ to $\mathrm{N}=5000$. The red line is a fit of the form $\mathrm{y}=0.975852 \mathrm{x}+0.00223295$

contains 6 extra positive disclinations due to Euler's theorem.

\section{Cluster Energies}

In this section we compare the scaling of the energy as given in section III.C with the energies found by simulated annealing.

Having identified the electrostatic and correlation energies as the first two leading order contributions to the energy of the cluster, which scale as $N^{2}$ and $N^{1.5}$ respectively, we make the ansatz that the remaining terms in the series descend in powers of $\sqrt{N}$, thus

$$
\begin{aligned}
E_{\text {Smooth }} & =\frac{\pi}{4} \frac{N^{2}}{R}+\left(\alpha-\beta \triangle \sqrt{\frac{1}{2 \pi}}\right) \frac{N^{\frac{3}{2}}}{R} \\
& +\kappa_{3} \frac{N}{R}+\kappa_{4} \frac{N^{\frac{1}{2}}}{R}+\kappa_{5} .
\end{aligned}
$$

The second term is divided into two parts: $\beta_{\triangle}$ is the correlation energy if the lattice is strictly triangular everywhere and the LDA applies; the term containing the parameter $\alpha$ is a correction to this assumption.

We now compare the scaling of the energy given by Eq. (30) with the energies found by simulated annealing. Since the electrostatic and correlation/surface terms dominate for large systems, it is natural to ask what influence the remaining terms may have, so we plot

$$
\frac{\Delta E}{N^{\frac{3}{2}}}=\frac{1}{N^{\frac{3}{2}}}\left(E-\frac{\pi}{4} \frac{N^{2}}{R}+\beta_{\triangle} \sqrt{\frac{1}{2 \pi}} \frac{N^{\frac{3}{2}}}{R}\right)
$$

against $N^{-\frac{1}{2}}$, see Fig. 7 From the intercept we find $\alpha=0.00223295$ and from the gradient $\kappa_{3}=0.975852$. Let us replace the coefficients multiplying the $N^{1.5} / R$ term in Eq. (30) with

$$
\kappa_{2}=\sqrt{\frac{1}{2 \pi}} \beta_{o}=\alpha-\sqrt{\frac{1}{2 \pi}} \beta_{\triangle},
$$

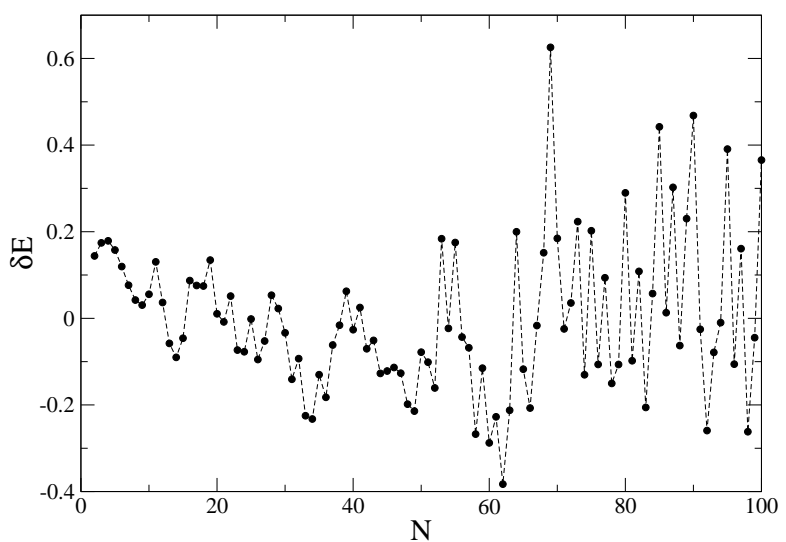

FIG. 8: A plot showing the difference between the energy for small clusters found by simulations and the smooth part of the energy.

where $\beta_{o}=3.915436$ is the 'observed' value of $\beta$. It differs from $\beta_{\triangle}$ by approximately $0.15 \%$. This might be due to a failure of the LDA approximation, but it might also be due to the fact that we are almost certainly not obtaining the ground states for each value of $N$, which has uncertain consequences for the accurate determination of the coefficient $\kappa_{2}$.

There is no appreciable bending of the curve shown in Fig. (7), which suggests that the remaining terms in Eq. (30) are insignificant in the large $\mathrm{N}$ limit. (The coefficients $\kappa_{3}, \kappa_{4}$ and $\kappa_{5}$ were actually determined by using a curve fitting package in the range $N=2$ to 100). Furthermore as all the data points lie almost perfectly on a straight line, we conclude that the numerical results are fairly reliable. In fact for large systems, this plot often enabled us to determine which clusters generated by the simulated annealing algorithm required further annealing. These were the clusters for which the data points were slightly above the fit plotted in Fig. 7.

Having determined the form of the "smooth" part of the energy we can determine the fluctuating part of the energy, which is defined as $\delta E=E-E_{\text {Smooth. }}$. The results are shown in Fig. 8, Some clusters have a particularly low value of $\delta E$ such as $N=34,49$ and 62 . These clusters are known as "magic number states" and posses a high degree of geometrical symmetry. To illustrate this point Fig. (9) shows a Delaunay triangulation of the system with 62 and 69 charges, which correspond to the lowest and highest points, respectively, in Fig. (8). 


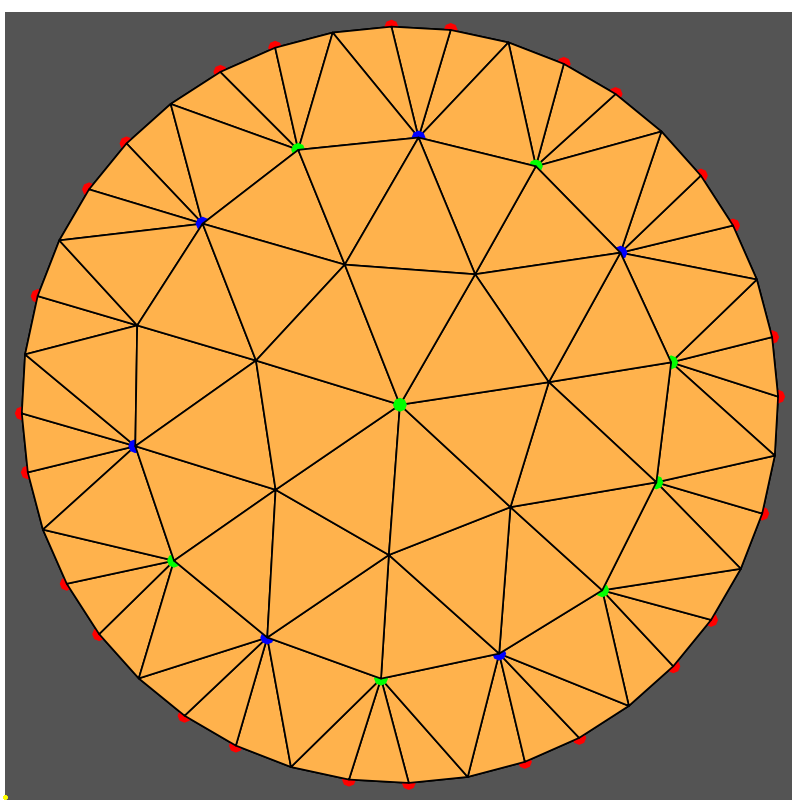

62 Charges

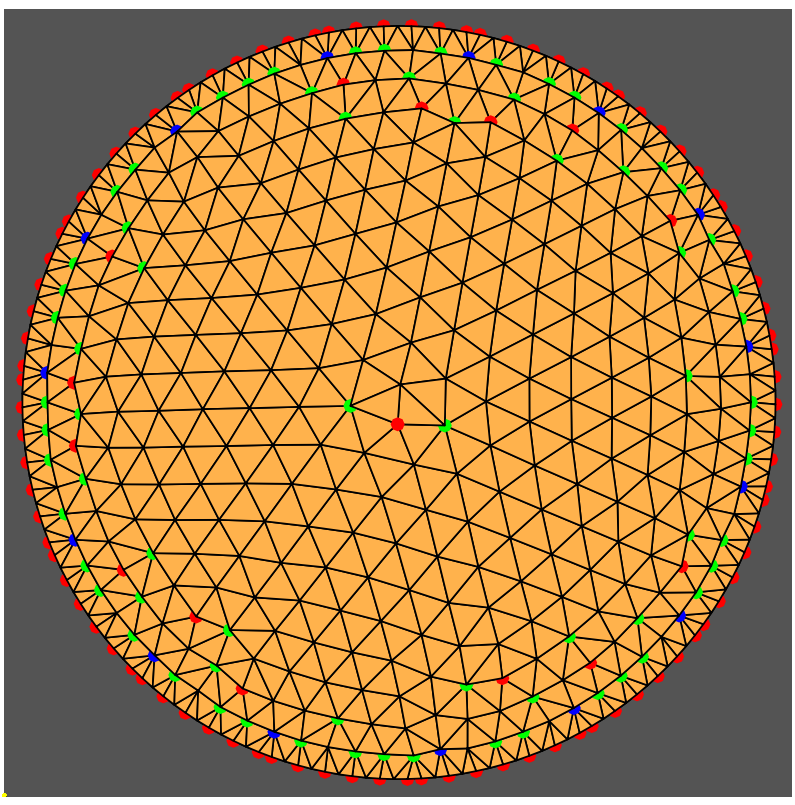

500 Charges

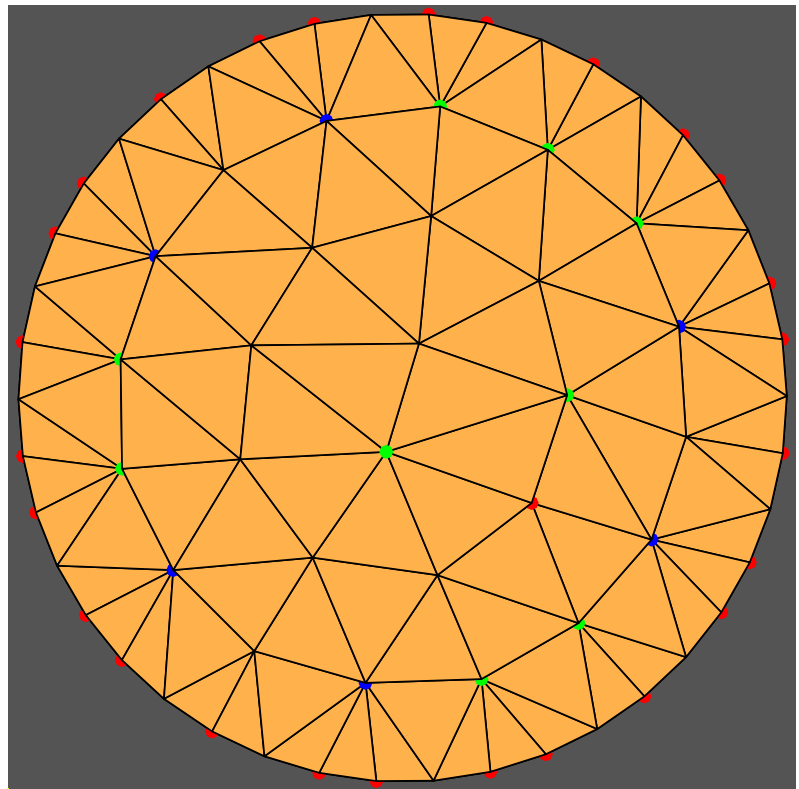

69 Charges

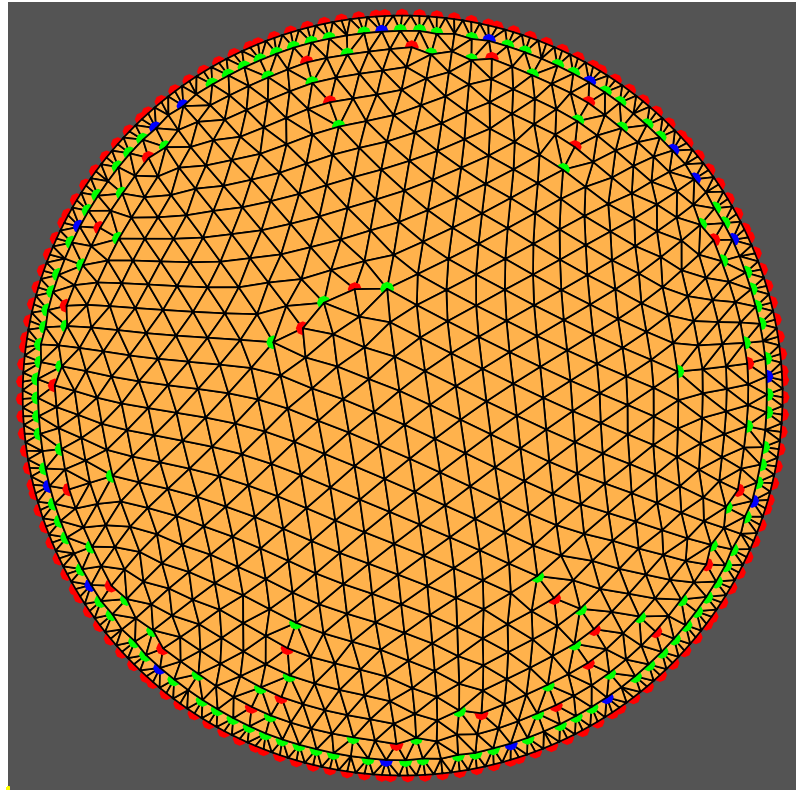

1000 Charges

FIG. 9: Clusters with a hard wall confining potential. The cluster with $N=62$ has the lowest measured value of $\delta E$, while the cluster with $N=69$ has a particularly high value. This is because the $N=62$ cluster possesses a high degree of circular symmetry, which is commensurate with the hard wall boundary. The lack of circular symmetry in the $N=69$ cluster is evident from the off-center negative disclination. In addition the boundary imposes a shell-like order towards the edge of the cluster, which is indicated by the presence of a large number of seven and eight coordinated disclinations. For larger systems the shell-like order is confined to within a few lattice layers of the edge, the lattice interior is composed of a triangular lattice with some charged grain boundaries. 


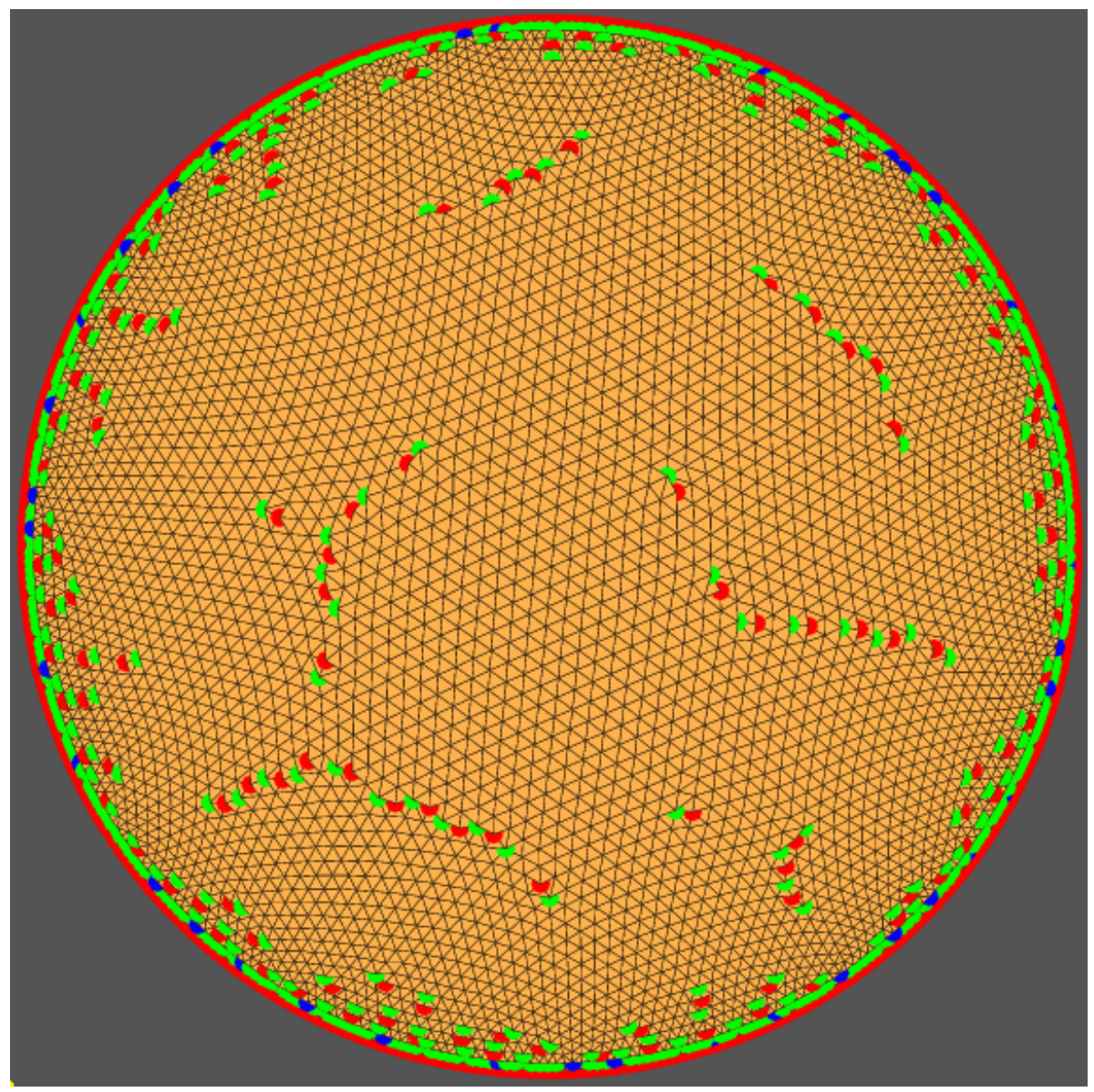

5000 Charges

FIG. 10: Clusters with a hard wall confining potential. The remarkable feature of this system is the arched like structure towards the edge. This curvature is due to an excess of negative disclinations in the lattice interior. Most of the charged grain boundaries appear to be located in the region between the relatively undistorted triangular lattice and the arches. We believe that the grain boundaries act to screen off the two incommensurate regions.

\section{Small, Medium and Large Clusters}

Small clusters, those which contain less than 100 particles, are dominated by the circular hard wall boundary. In particular, clusters containing less than 50 charges show no hint of hexagonal order. Instead the charges are arranged in concentric shells, where many of the charges have 7 or 8 nearest neighbors. We identify this type of ordering as "shell-like"; it is present at the edge of all the systems. For systems containing more than 50 particles, a region of the cluster free from the influence of the hard wall boundary begins to emerge; this region looks like a triangular lattice. At this point there is a competition between shell-like order and the emerging hexagonal order. The clusters with the lowest energy are the ones which can satisfy both simultaneously. Both $N=62$ and $N=69$ are clusters which contain a region where most of the charges have six nearest neighbors. The charges near the center in both clusters can be considered to be part of a disclination as the central charge has a different coordination number compared to its neighbors. Of all the clusters, $N=62$ has the lowest value of $\delta E$ while $N=69$ has the highest. The difference is that for $\mathrm{N}=69$ the disclination is off-center which breaks circular symmetry while in the case of $N=62$, the disclination is perfectly in the center of the cluster and is surrounded by a ring of charges which have six nearest neighbors.

For the medium sized clusters, i.e. $100 \leq N \leq 1000$, the influence of the hard wall boundary on the cluster is contained within a narrow annular region at the edge. This again is the shell-like region and contains a large number of seven and eight coordinated disclinations, which have the effect of destroying the hexagonal order. On the other hand, the internal region of the lattice is comprised of a relatively undistorted triangular lattice lattice. The lattice interior contains an excess of negative disclinations. However, for every excess negative disclination in the interior there is a compensating posi- 


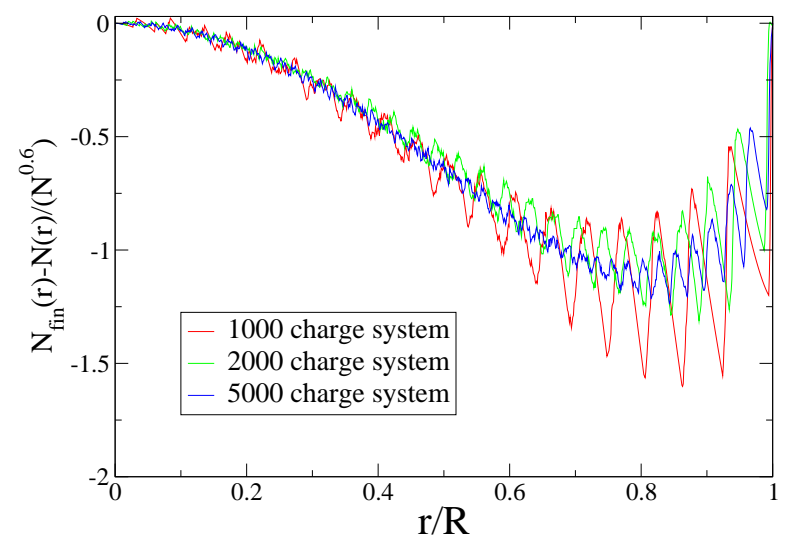

FIG. 11: Plot of $\Delta N$ for the three largest systems with a harmonic confining potential, scaled by $N^{0.6}$ versus distance $r$ from the center.

tive one on the lattice edge. Using the rule that a charge with 7 or 8 nearest neighbors is a disclination with topological charge -1 and -2 respectively, while a charge with 5 nearest neighbors has charge +1 , the total topological charge of all the clusters was found to be +6 . The 6 positive disclinations due to Euler's theorem are also located on the lattice edge, hence Euler's theorem is satisfied.

Also with increasing system size isolated disclinations become less common and are replaced by small topologically charged grain boundaries; these are chains of alternating positive and negative disclinations, which contain in total one excess negative disclination. As shown in Fig. (14), the alternating Burgers vectors of the charged grain boundary cancel out and the overall arrangement constitutes a disclination.

Large clusters are those which contain more than 1000 charges, see Fig. (10). In this regime, the interior contains large areas of lattice separated by charged grain boundaries, which are numerous and long. As will be shown in Section V.A, excess disclinations of one sign in the lattice interior generate lattice curvature, the effect of which is to bend the lattice lines into a series of arches. This can be seen with great clarity in the $N=5000$ system. For this system, the bending is sufficiently strong to make the arched structure incompatible with the ordinary triangular lattice. It would seem that the grain boundaries arrange themselves to screen off the two regions.

\section{B. Harmonically Confined System}

In this Section we consider a 2D cluster of charges confined by a harmonic confining potential. This system has been studied in considerable detail by Koulakov and

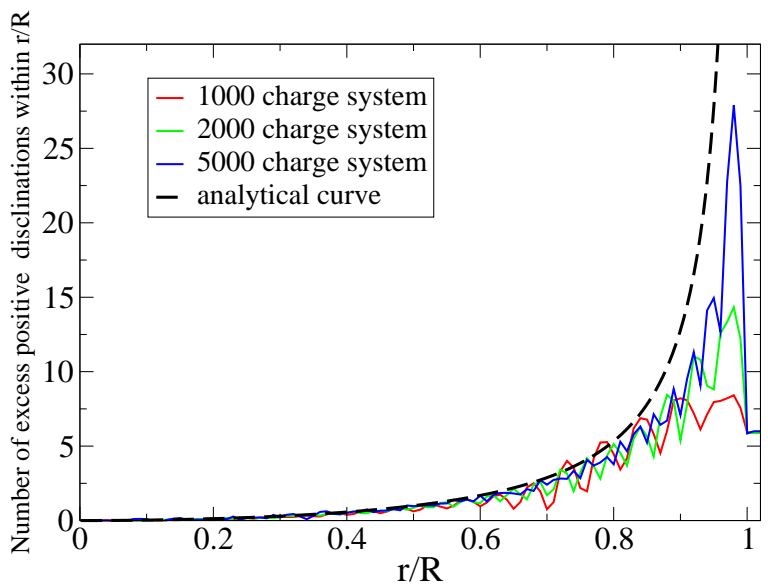

FIG. 12: A plot of the number of excess positive disclinations within a distance $r / R$ where we use the rule that a five coordinated and a seven coordinated disclination count as a single positive or negative disclinations respectively. Results for clusters containing 1000, 2000 and 5000 clusters are colored red, green and blue respectively. The analytical curve is given by the black dashed line. Note that at the edge of the cluster the number of excess positive disclinations falls to +6 . Unlike the number of excess disclination in the hard wall system, the number in this system lags behind the continuum value significantly.

Shklovskii, see [3] and [2], and here we expand on their work.

As for the system with the hard wall confining potential, the actual density differs from the continuum limit result by subdominant contributions which are difficult to quantify. Plotting $\Delta N=N_{f i n}(r)-N^{P}(r)$ for the three largest systems shows that there is a deficiency of charge in the system interior which is compensated for by a shell of excess charge at the edge of the system. A good collapse of the data for different values of $N$ was found as in when we plotted

$$
\frac{\Delta N}{N^{0.6}}=\frac{N_{f i n}(r)-N^{P}(r)}{N^{0.6}},
$$

but the origin of this correction with the power 0.6 is a mystery to us.

In the continuum limit, the density of excess disclinations and the density of the Burgers vector for this and the hard wall systems are the same except for the sign. It follows that the number of excess disclinations within a given radius is the same for the two systems. A comparison with our numerical simulations is given in Fig. (12).

Koulakov and Shklovskii demonstrated that provided the cluster is small $(N \leq 150)$ then there exist certain magic number states which contain only 6 fivecoordinated disclinations demanded by Euler's theorem. 


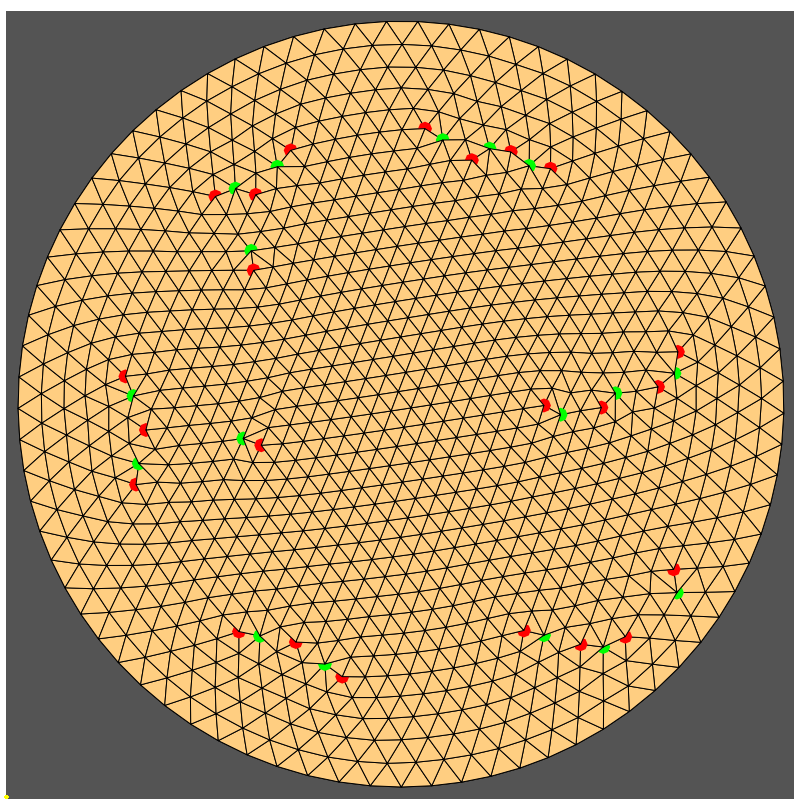

1000 Charges

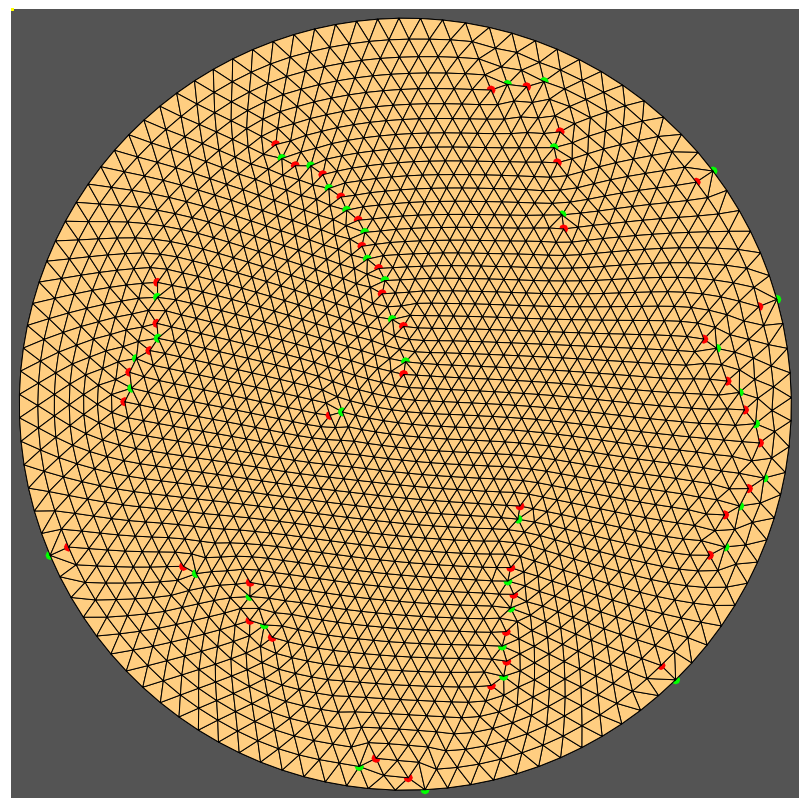

2000 Charges

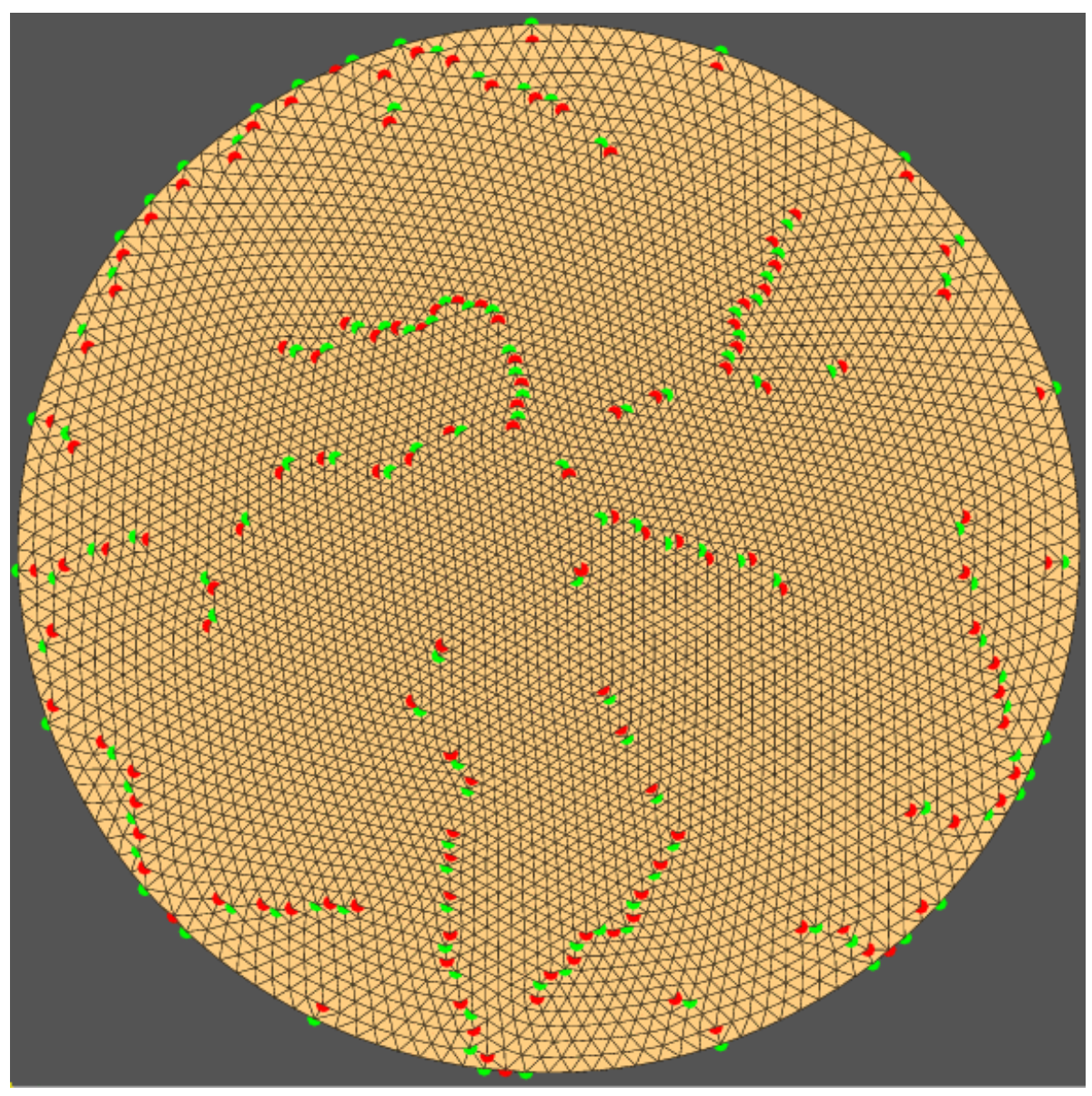

5000 Charges

FIG. 13: Clusters with a harmonic confining potential. 1000 charges: This cluster contains both charged grain boundaries and also some isolated dislocations. These dislocations are not involved in screening, but serve to relax the strain energy. 2000 charges: With increasing system size, the charged grain boundaries continue to grow in length. Also the isolated dislocations become more numerous, especially near the edge of the system. Note these dislocations are oriented with the 5 coordinated disclination pointing towards the center of the system. There is a rapid rise in the number of positive disclinations just before the lattice edge which are canceled by the negative disclinations close to the edge. 5000 charges: For very large systems, the additional dislocations required to accommodate the changing density form uncharged grain boundaries (i.e grain boundaries with no over-all topological charge). Clear examples of such grain boundaries can be seen towards the edges of the cluster. 
These disclinations are not right on the lattice edge but are close to it. Beyond this limit $(N \geq 150)$ the disclinations are always accompanied by a screening cloud of dislocations. It was further shown that provided the cluster is not too large $(N \leq 700)$ then the only defects in the lattice are the disclinations and their screening clouds, which together form 6 small separate topologically charged grain boundaries. In fact provide that $N \leq 700$, the lattice can be divide into an inner region and an outer region, where the boundary between the two is given by the radius at which the charged grain boundaries are located. The inner region is a largely undeformed triangular lattice while in the outer region the lattice lines are curved.

Beyond this limit $(N \geq 700)$ dislocations not associated with screening begin to appear, these dislocations are present in order to ensure that Eq. (23) is satisfied, consequentially the lattice cannot be divided so neatly into two separate regions [3].

The numerical work of Koulakov and Shklovskii only dealt with systems containing less than 700 charges. Our work is concerned with the behavior of the system as the cluster grows beyond this limit. An examination of the cluster containing 1000 charges, see Fig. (13), shows that in addition to the topologically charged grain boundaries the system also contains a few isolated dislocations. These dislocations are orientated so that the 5 coordinated disclination points towards the center of the lattice while the 7 coordinated disclination points radially outwards. With increasing system size the general trend is that the screening cloud around the disclinations continue to grow in length. In addition the isolated dislocations become increasingly numerous, see the cluster containing 2000 charges in Fig. (13). Eventually in addition to isolated dislocations we can observe uncharged grain boundaries (i.e extended chains consisting of alternating disclinations but which have no overall topological charge), see the system containing 5000 charges, Fig. (13).

Even though the continuum disclination charge density is the same for the hard wall system and the system with harmonic confinements, nevertheless there is a remarkable difference between the two in their approach to the continuum limit with increasing system size. Compared to the hard wall system, the number of disclinations in this system is far fewer. From Fig. (12) we can see that the number of disclination within a given radius lags behind the continuum value, while the opposite is true in the hard wall system. For instance, for 5000 charges in a harmonic trap the number of excess disclinations at the edge is about 25, while in the hard wall case this is 400 . Even though the curvature of lattice lines in the continuum limit (which is given by the density of Burgers vector) is the same for the two systems, the curvature of the lattice lines appears to be far less pronounced for the system with harmonic confinement. This may be due to the lack of disclinations, since the curvature depends on the number of disclinations enclosed within a radius, see

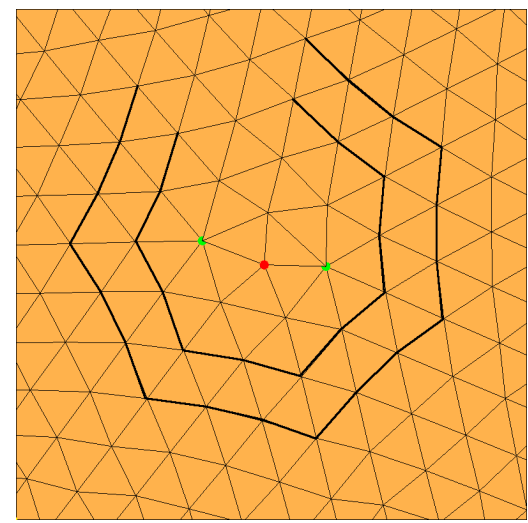

FIG. 14: A close up of a small grain boundary with a total topological charge of -1 . The arrangement can also be thought of as a pair of dislocations with opposite Burgers vectors which cancel. Drawing a Burgers circuit around the grain boundary leads to a closure failure which, like a disclination of charge -1 , increases with distance from the center.

\section{Eq. (38).}

Unlike the hard wall system, which requires an excess of 7 coordinated disclinations in the interior, the harmonic system already contains six free 5 coordinated disclination due to Euler's theorem. In the case of the hard wall system these topologically induced disclinations are pushed to the edge of the system while in the harmonic case these disclinations sink into interior and help in accommodating the non-uniform density. In contrast to the hard wall system, the density of free disclinations, given by $s(r)$ in Eq. (22), cannot be ignored. From Fig. (12) it can be seen that up to $r=0.8 R$ the total disclination charge does not exceed +6 . Thus up to this point the mechanism by which the lattice adapts to the decreasing density towards the edge of the system depends on the arrangement of these free disclinations. In order for the total disclination charge to match that given by Eq. (23), dislocations arise to screen the disclinations. These screening dislocations have the effect of smearing out the disclination charge. To explain what we mean by this, consider the following qualitative argument. To define a disclination we must be able to draw a Burgers circuit around it. The smallest Burgers circuit around a single disclination has a radius equal to the local lattice spacing. This then defines the minimum size of the defect. By screening the disclination to form a charged grain boundary, of the type shown in Fig. (14), the radius of the Burgers circuit needed to enclose the object becomes larger, hence the size of the defect is increased. However, the total disclination charge enclosed is still the same. Thus the total density of disclination charge, i.e. $\tilde{s}(\mathbf{r})$, is reduced. Or to put it another way, unlike an isolated disclination, which is a single point in the lattice, the charged grain boundary is an extended object. However, the total charge of the grain boundary is still \pm 1 . Thus we can consider this charge to be 
smeared out over its length.

Beyond $r=0.8 R$ there is a sharp increase in the number of dislocations, which for the largest systems condense into uncharged grain boundaries. These dislocations are oriented such that the 5 coordinated disclination points inwards while the 7 coordinated disclination is on the lattice edge. Thus towards the edge of the lattice there is a sudden jump in the number of excess positive disclinations followed by an equally sudden fall. Perhaps as the number of charges in the system is increased further, these dislocations might unbind so that the 7 -coordinated disclinations are pushed to the lattice edge while the 5 -coordinated disclinations remain in the interior.

\section{DISCUSSION}

\section{A. Lattice Curvature}

The most remarkable feature of the numerical simulations is the bending of lattice lines towards the edge of the cluster; which is particularly strong in the larger systems - see for example Fig. (10). This bending is due the fact that the lattice contains an excess of dislocations of one sign. The original argument explaining this phenomena was given by Nye [16], who showed that the curvature of the lattice is equal to the Burgers vector density. However, Nye's exposition assumes that the lattice spacing remains constant everywhere. Blindly applying Nye's result to our system would predict that the lattice lines bend in the opposite direction. With a slight modification we can adapt Nye's argument to explain lattice curvature in crystals with a changing density.

Consider a square section of lattice pqrs of dimension $\Delta r$ as shown in Fig. (15. A), the lattice has a constant density everywhere and so there are an equal number of lattice lines crossing each side of the square. If on the other hand the density is increasing in the $\mathrm{r}$ direction it means there must be more lattice lines crossing the side $s r$ than $p q$. Drawing the Burgers construction around the square leads to a closure failure. Consequentially the square must contain an excess of edge dislocations of one sign, the sum of their individual Burgers vector being equal to the total Burgers vector B. This situation is shown is Fig. (15.B), it can be imagined that the total Burgers vector is split into two equal parts and all the dislocations are contained within the triangles $p$ ' $t$ 's' and $q$ ' $u$ ' $r$ ' - both of which subtend an angle of $\theta / 2$ - it follows that

$$
\frac{\theta}{2} \approx \frac{\frac{1}{2} n B}{\Delta r}
$$

where for small values of $\theta$ we can ignore higher order terms. It is useful to imagine that the original lattice has been plastically deformed into the quadrilateral $p$ ' $q$ 's' $r$ '. However, this picture is misleading as none of the lattice lines which were originally parallel to $p s$ and $q r$ suffer a change in length; thus the state shown in Fig. (15,B) is for illustrative purposes only. The true state of the deformed lattice is shown in Fig. (15,C). By mapping the sides $p q$ and $s r$ of the original square onto the circular arcs $p$ ' $q$ ' and $s$ 'r', all of the lattice lines originally parallel to $p s$ and $q r$ remain of length $\Delta r$ - at the expense of being no longer parallel to each other. To find the curvature $k$ of the bending of the arcs, let the length of the lines $o^{\prime} p^{\prime}$ and $o^{\prime} q^{\prime}$ be equal to $\mathrm{L}$. We use the relationship

$$
\Delta r=\frac{\theta}{k}
$$

where $k=1 / L$. Upon substituting for $\theta$ from Eq. (34) and taking the continuum limit we have

$$
\mathbf{k}(\mathbf{r})=\lim _{\Delta r \rightarrow 0} \frac{n B}{(\Delta r)^{2}}=\mathbf{b}(\mathbf{r})
$$

Hence the curvature is equal to the Burgers vector density. If on the other hand the lattice density is decreasing in the $r$ direction then we expect the sense of curvature to be reversed. Furthermore, for lattice lines not parallel to the local Burgers vector, then the curvature of the lattice line $\mathbf{k}_{\mathbf{o}}$ depends on the angle $\gamma$ it makes with the local Burgers vector [16]

$$
\mathbf{k}_{\mathbf{o}}(\mathbf{r})=\mathbf{k}(\mathbf{r}) \cos \gamma
$$

The next logical step is to show that the lattice curvature in the systems which we have simulated is given by the Burgers vector density. Consider the deformed hexagon shown in Fig. (16) where the direction of $\mathbf{b}$ is marked by an arrow. We expect the curvature of the lattice lines to be described by Eq. (36). To make a connection with our simulations, for the $N=5000$ system with a hard wall boundary shown in Fig. (10), each hexagon is decomposed into three arcs, each of which can be further decomposed into three points as in Fig. (16). By fitting the points to the equation of a circle we determined the curvature of each arc. We assume that each arc yields the curvature of the lattice at the center of the hexagonal cell. Depending on the radial distance of the cell from the center of the lattice we expect this curvature to have any value between 0 and $|\mathbf{b}(\mathbf{r})|$ (depending on the orientation of the lattice line with respect to the local Burgers vector density field). For the hard wall systems with 5000 charges, Fig. (17) shows the curvature of each such arc against radial distance; also plotted for comparison is the Burgers vector density given by Eq. (19). Ideally the curvature ought not to exceed the limit set by Eq. (19), however, this is not possible in reality as lattice lines close to disclinations suffer much greater curvatures than Eq. (19) would allow. From the general similarity of the curvature data and Eq. (19) we conclude that the cause of the bending of lattice lines is indeed due the plastic deformation of the lattice.

Next we wish to make the connection between curvature as defined by the work of Nye to some well-known results about parallel transport of a unit vector about a 


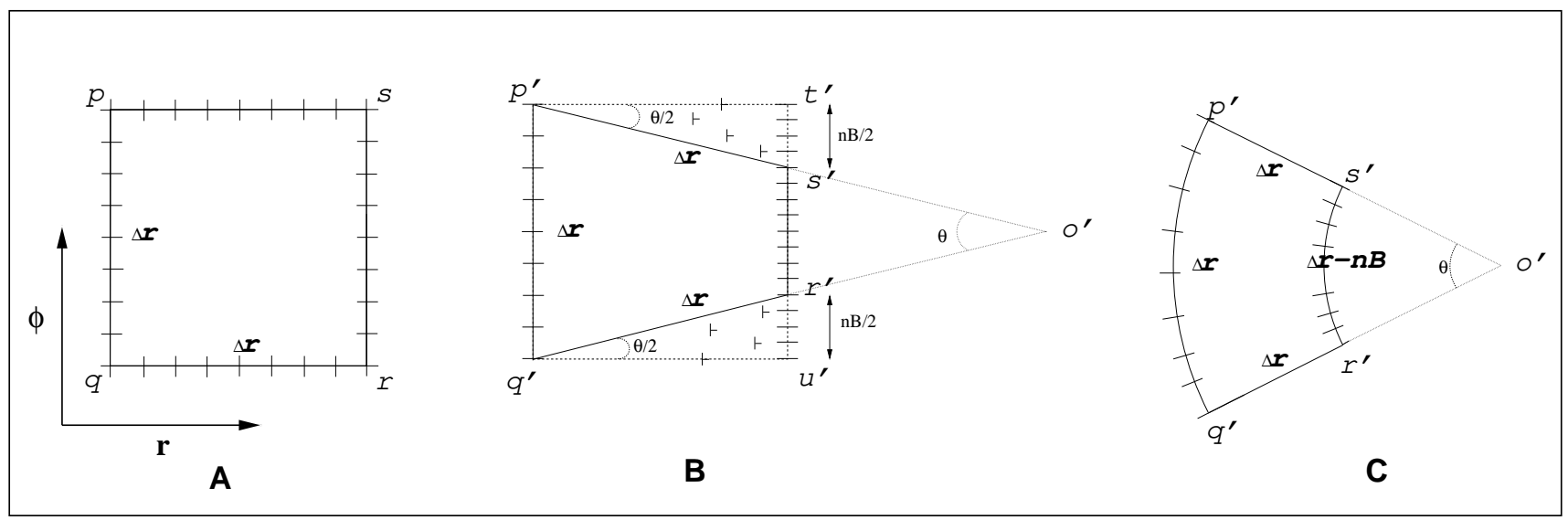

FIG. 15: $[A]$ : An undeformed lattice of dimensions $\Delta r .[B]$ : A lattice of increasing density, where the change in lattice density depends only on the $\mathrm{r}$ direction. In effect the original square lattice has been plastically deformed into the quadrilateral $p$ ' $q$ ' $r$ ' $t$ '. This diagram is geometrically incorrect as only the lines originally parallel to pq and sr should suffer a change of length, while those originally parallel to $p s$ and $q r$ should remain of the same length. $[C]$ : The true deformed state of a lattice with increasing density, the lattice lines $p q$ and $s r$ are deformed into the circular arcs $p$ ' $q$ ' and $s$ ' $r$ '. This state is geometrically correct as all of the lattice lines which were originally parallel to $p s$ and $q r$ are still of the same length. Note this construction only tells us how to calculate the curvature of lattice lines which were originally parallel to $\mathbf{B}$

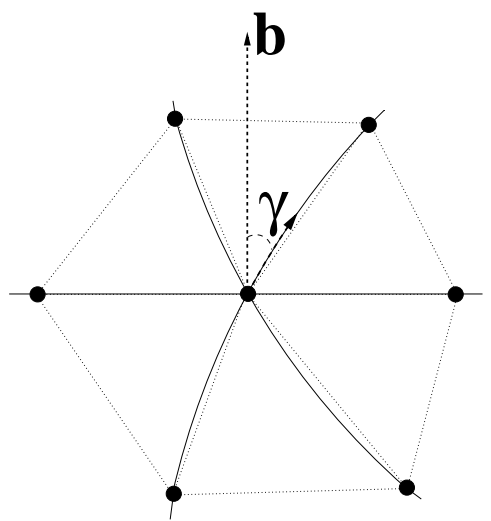

FIG. 16: This diagram shows a hexagonal cell in a lattice with a non-zero Burgers vector density field $\mathbf{b}$. The hexagonal cell can be decomposed into three arcs which cross the center of the cell, if the arc makes an angle $\gamma$ with the vector $\mathbf{b}$ then we expect its curvature to be given by $|\mathbf{b}| \cos \gamma$. Thus as shown in this diagram the horizontal arc which is perpendicular to b has no curvature.

disclination [17]. If a unit vector is transported on a lattice along some path enclosing disclinations, then when the vector returns to its original position its orientation will have changed. The total change depends on the number of disclinations enclosed and in a triangular lattice must be a multiple of $\pi / 3$. To show this connection we can invert Eq. (21) to express the density of the Burgers vector in terms of the disclination charge enclosed

$$
b_{\phi}(r)=\frac{1}{r} \int_{0}^{r} \tilde{s}\left(r^{\prime}\right) r^{\prime} d r^{\prime}=\frac{1}{2 \pi r} \int_{0}^{r} \int_{0}^{2 \pi} \tilde{s}\left(r^{\prime}\right) r^{\prime} d r^{\prime} d \phi .
$$

If as before we let $\Sigma(r)$ be the total disclination charge within a disk of radius r, then using Eq. (35) we can write Eq. (37) as

$$
k_{\phi}(r)=\frac{\Sigma(r)}{2 \pi r} .
$$

This equation expresses the fact that the curvature at a distance $r$ from the center of the system depends on the total disclination charge enclosed within a disk of radius r. For a given curve the curvature is defined as the rate of change of the angle of its tangent vector $\omega$, thus for the circular path enclosing a disclination charge $\Sigma(r)$ we have

$$
\frac{1}{r} \frac{d \omega(r)}{d \phi}=k_{\phi}(r) .
$$

To find the total change in the angle of the tangent vector we integrate over the length of the circular path giving,

$$
\omega(r)=\int_{0}^{2 \pi} r k_{\phi}(r) d \phi=\Sigma(r),
$$

where in a real lattice the disclination charge is quantized. This result explains why the orientation of the lattice cells in images such as Fig. (10) is observed to rotate upon traversing a circular path centered on the origin.

It should be noted that as long ago as 1955 the geometry of imperfect lattices had been developed by Kondo 


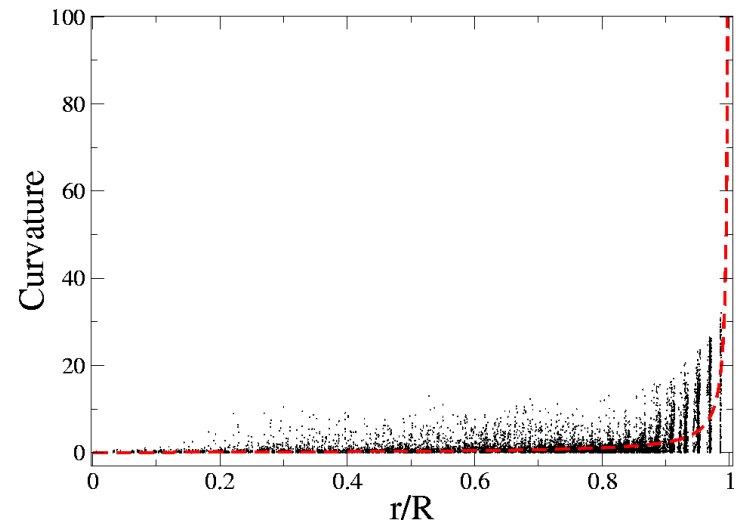

FIG. 17: Curvature of lattice lines in the system with 5000 charges in a hard wall confining potential. As discussed in the text, each hexagonal cell can be decomposed into a set of 3 arcs; for each such arc the curvature is computed and plotted against the radial distance of the parent hexagon this is shown by the black dots. Theoretically the maximum curvature that an arc can have is given by $|\mathbf{b}(\mathbf{r})|$ which is shown by the red dotted line. Thus depending on the orientation of the lattice line, the curvature ought to range from 0 to $|\mathbf{b}(\mathbf{r})|$. The fact that the lattice curvature exceeds $|\mathbf{b}(\mathbf{r})|$ for some arcs is due to the fact that close to the core of a defect the curvature becomes much larger than that set by the continuum limit calculation.

and co-workers into non-Riemann differential geometry [18]. By analogy to general relativity, one can think of an undeformed lattice as a region of flat space, which becomes warped in the presence of defects. The authors demonstrated that dislocations and disclinations generate torsion and curvature respectively.

\section{B. Conformal Crystals}

The systems we have discussed thus far have been constructed using optimization algorithms to generate ground state configurations. Surprisingly there exists an unusual class of 2D lattices which have a non-uniform density but which can be constructed by a purely analytical method. These structures are known as conformal crystals [19]. As the name suggests the positions of the lattice sites can be obtained in the image plane by applying a conformal transformation to a regular lattice in the z-plane.

An example of a conformal lattice with circular symmetry is shown in Fig. (18). As evident from the way in which the lattice lines curve towards the edge and the increasing density, there is a resemblance between the conformal crystal and the clusters studied in Section IV. Initially it was hoped that these conformal crystals might help explain the origin of the lattice curvature observed in the other 2D systems, but this did not turn out to be the case. In this section we show that conformal crystal can be regarded as a giant disclination.
Originally the idea of conformal crystals was used to describe a structure formed by a cluster of mutually repelling magnetized spheres dubbed "gravity's rainbow". In an experiment, metallic spheres were confined to a thin rectangular box which is placed in a magnetic field, the field induces a magnetic moment in each of the spheres which causes them to repel. Under the action of gravity the spheres crystallize into a lattice with non-uniform density, which consisted of a series of arch-like structures. The authors suggested that the unusual lattice could be obtained by a conformal transformation of a regular triangular lattice [20].

Consider for example a regular triangular lattice in the z-plane such as that shown in Fig. (18- left). By applying an analytical transformation $w=f(z)$ the corresponding coordinates in the w-plane are

$$
u+i v=f(x+i y),
$$

where $w=u+i v=r e^{i \theta}$ and $z=x+i y=r^{\prime} e^{i \phi}$. In the case of $f(z)=z^{\frac{1}{2}}$ the result of the transformation is shown in Fig. (18). This transformation belongs to a set of transformations

$$
w=C z^{\frac{1}{\chi}} \text { or } w=e^{C z},
$$

which yield conformal lattices of circular symmetry. Here we shall only concern ourselves with the first of these transformations.

Conformal transformations have three important features. Firstly they are locally angle preserving (isogonal) transformations. This means that upon mapping an infinitesimal hexagonal lattice cell in the $\mathrm{z}$ plane to the image plane, the cell will still have all its internal angles equal to $\pi / 3$. It is important to note that because of the local nature of the angle preserving property this is only strictly true for an infinitesimal lattice cell. For a real lattice with a well-defined lattice spacing, the mapping distorts the shape of the hexagonal cells. This distortion is stronger towards the center of the lattice than the edge, see Fig. (18) for an illustration of this. At the origin the conformality of the transformation breaks down completely. In the case of the transformation $w(z)=z^{1 / 2}$, instead of preserving angles, the angle of $\pi / 3$ at the origin is halved in the image plane. No matter how small the lattice cell enclosing the center in the z-plane is made, this breakdown of conformality will remain. If the conformality of an otherwise conformal mapping breaks down at a particular point, then that point is called a critical point of the mapping. The critical points of a conformal transformation exist at any point where either $|d w / d z|$ or its inverse is equal to zero 21]. Secondly, a lattice with constant density $\rho_{z}$ in the z-plane will have a density [19]

$$
\rho_{w}=\rho_{z}\left|\frac{d w}{d z}\right|^{-2},
$$

in the w-plane. Thirdly, it was shown that if the transformation is conformal then the density of lattice points in 

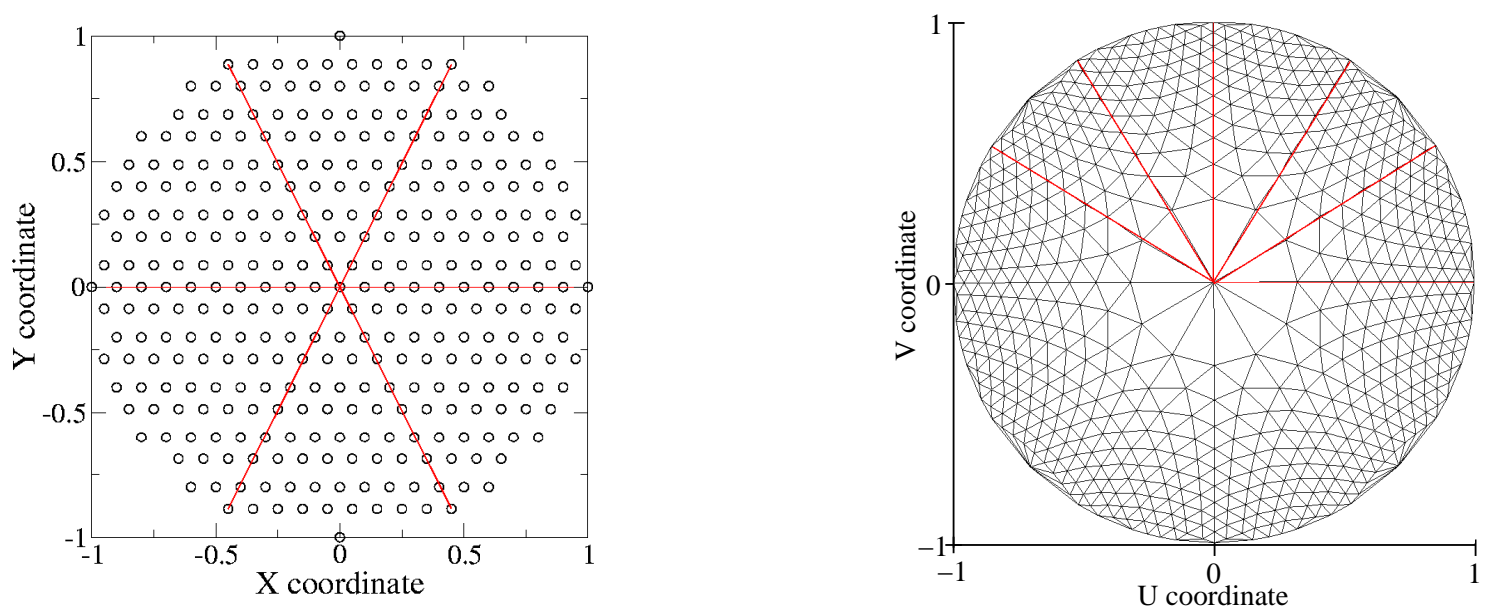

FIG. 18: Left: a circular cut out of a triangular lattice in the $\mathrm{z}$ plane, where the angle between each successive red line is $\pi / 3$. Right: the effect of the transformation $w=z^{\frac{1}{2}}$ in the image plane, where the lattice has been produced by allowing the range of the $\mathrm{z}$ plane to extend to $\phi<4 \pi$. Conformal transformations are locally angle preserving transformations, therefore an infinitesimal hexagonal lattice cell in the $\mathrm{z}$ plane is not deformed upon mapping to the image plane. However, this angle preserving property of the transformation breaks down at certain points which are known as critical points. For the transformation $w=z^{\frac{1}{2}}$ this occurs at the origin, where instead of preserving the angle $\pi / 3$, it is halved. Note that the lattice cells are more distorted closer towards the critical point.

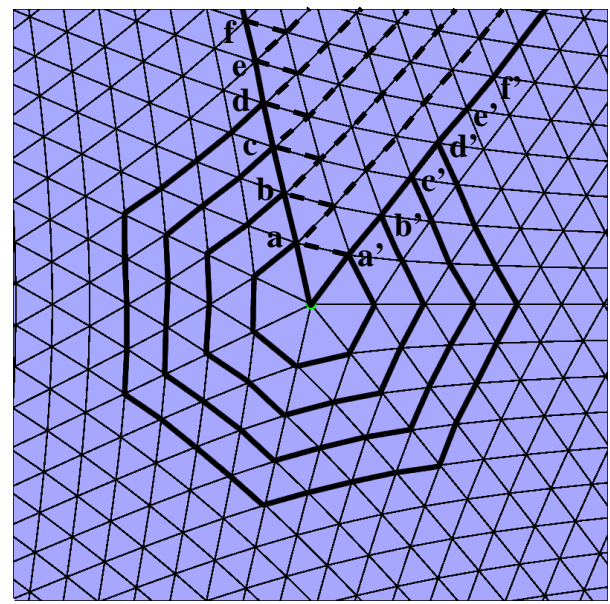

FIG. 19: A conformal lattice generated by the transformation $w=z^{6 / 7}$ which is similar to a disclination with charge -1 . Also shown are a series of Burgers circuits enclosing the central point.

the image plane is constrained by the following condition [20]

$$
\nabla^{2} \ln \rho(r)=0,
$$

which interestingly is also the condition that the lattice has no disclination charge induced by a varying density, see Eq. (21).

Thus a circularly symmetric conformal crystal such as the one shown in Fig. (18) is locally hexagonal but has curvature. It has an increasing density and except for the point at the center has no apparent internal defects (by which we mean that a triangulation only shows internal points which have six nearest neighbors). Yet the framework developed in Sections 2.3 and 2.4 demonstrates that a change in the lattice density must be accompanied by lattice defects, which in turn are responsible for curvature. How are these two seemingly conflicting statements to be resolved? A clue is provide by the point at the center of the lattice with the anomalous coordination number.

Consider the conformal lattice generated by the transformation

$$
w(z)=z^{\frac{6}{7}},
$$

which is shown in Fig. (19). Superimposed on top of the conformal lattice are a number of Burgers circuits which enclose the central point. Whereas previously we were dealing with a continuum, in which the defects were assumed to form a gas throughout the system, here there is only a single defect at the center of the system. Since there are no other defects, any Burgers circuit which does not enclose this central point will close. As shown the circuits start at a,b... and end at a',b'..., in each case there is a closure failure, which increases with distance from the central point; indeed the successive Burgers circuits trace out a wedge. Thus we propose the central point in a conformal lattice can be thought of as a "disclination". The same rules which apply to a disclination also apply here, i.e. the wedge angle is quantized by the symmetry of the lattice and the wedge in turn can be decomposed into a series of half planes [11]. To see how a wedge can 
be decomposed into a series of half planes, note that the difference in the closure failure between any two successive Burgers circuits, such as aa' and bb', is always one lattice spacing, which implies that between a pair of circuits an extra half plane has been inserted at the points labeled a,b... The question of where the half planes have been inserted is arbitrary as it depends on where the Burgers circuits start and end. Thus as for a disclination the relationship given by Eq. (14) also holds for a conformal lattice. In the case of $w(z)=z^{\frac{6}{7}}$ (also for a -1 disclination) the wedge angle is given by $\Omega=\pi / 3$. By considering a series of circular concentric circuits whose origin coincides with the critical point of the transformation, it is possible to draw a series of Burgers circuits and calculate the Burgers vector density using the approach outlined in section III.B, in either case the result is still given by Eq. (17).

Since at the critical point the lattice ceases to be conformal, Eq. (41) is true everywhere except for the origin. This suggests that it can be written as

$$
\nabla^{2} \ln \rho(r)=\nu \delta^{(2)}(\mathbf{r}),
$$

where $\nu$ is an undetermined constant and $\delta^{(2)}$ is the two dimensional delta function. To find $\nu$, we are going to assume that the point at the center of the lattice is a disclination with charge $\tilde{s}(r)$. We have the following relationship between Eq. (42) and Eq. (21)

$$
2 \tilde{s}(r)=\nabla^{2} \ln \rho(r)=\nu \delta^{(2)}(\mathbf{r}) .
$$

Substituting $w(z)=C z^{\frac{1}{x}}$ into Eq. (40) gives

$$
\rho(r)=\rho_{z} \chi^{2} r^{\prime \frac{2(\chi-1)}{\chi}}=\rho_{z} \chi^{2} r^{2(\chi-1)},
$$

where we have used the relationship $r=r^{\prime \frac{1}{\chi}}$. Thus we can write Eq. (43) as

$$
2 \tilde{s}(r)=2(\chi-1) \nabla^{2} \ln r=\nu \delta^{(2)}(\mathbf{r}),
$$

Recognizing $\nabla^{2} \ln r=2 \pi \delta^{(2)}(\mathbf{r})$ as the two dimensional Green's function and canceling out the factor of 2, Eq. (45) becomes

$$
\tilde{s}(r)=2 \pi(\chi-1) \delta^{(2)}(\mathbf{r})=\nu^{\prime} \delta^{(2)}(\mathbf{r}),
$$

thus the central point of the conformal transformation has a disclination charge density of $\nu^{\prime}=\nu / 2=2 \pi(\chi-1)$. The total disclination charge contained within the disk can be found by simply integrating over its area, thus

$$
\begin{aligned}
\Sigma(r) & =\int_{0}^{R} \int_{0}^{2 \pi} \tilde{s}(r) r d r d \theta \\
& =2 \pi(\chi-1) \int_{0}^{R} \int_{0}^{2 \pi} \delta^{(2)}(\mathbf{r}) r d r d \theta \\
& =2 \pi(\chi-1),
\end{aligned}
$$

In the case of $\chi=2$ which corresponds to the transformation $w(z)=z^{1 / 2}$, the central point of the transformation

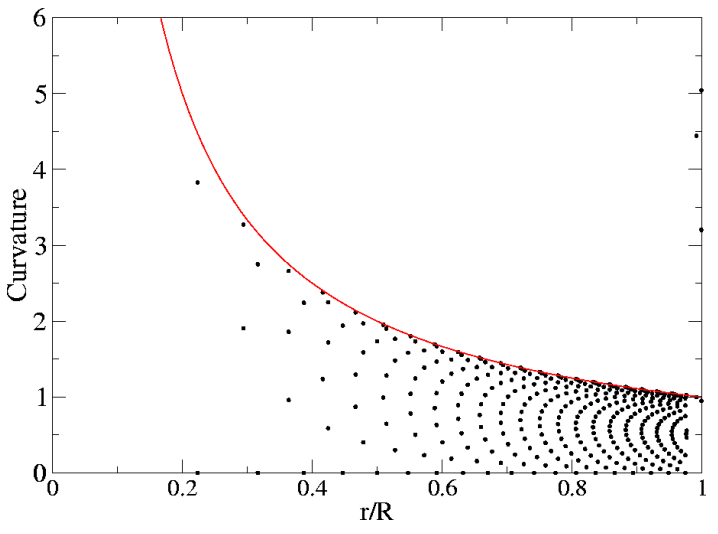

FIG. 20: Curvature of the lattice lines for a conformal crystal generated by the transformation $w(z)=z^{\frac{1}{2}}$. The red line gives the maximum curvature using Eq. (47). The black dots give the actual curvature of the lattice line, as calculated by the method outlined in Section 4.5.

has a disclination charge of $6(\pi / 3)=2 \pi$, i.e. a disclination consisting of 6 wedges, each of which subtend an angle of $\pi / 3$ in the $z$ plane.

This realization that the central point in a conformal lattice is actually a disclination can be used to calculate the lattice curvature. The conformal lattice shown in Fig. (18) was generated by applying the transformation $w=$ $z^{\frac{1}{2}}$ to a hexagonal lattice, the original lattice contained $N_{z}$ points within a disk of radius $\mathrm{R}$, thus $\rho_{z}=N_{z} / \pi R^{2}$, setting $\chi=2$ in Eq. (44) yields

$$
\rho_{w}(r)=4 r^{2} \rho_{z},
$$

Using the framework developed in Section 2.4 we know that the maximum curvature $k$ is related to the density of the Burgers vector by Eq. (35), thus

$$
k(r)=|\mathbf{b}(\mathbf{r})|=\frac{1}{2} \frac{d}{d r} \ln \rho_{w}(r)=\frac{1}{2} \frac{d}{d r} \ln 4 r^{2} \rho_{z}=\frac{1}{r},
$$

where we have used Eq. (17). On the other hand, using Eq. (14) with $\Omega=2 \pi$ gives $|\mathbf{B}(r)|=(2 \pi) r$. Inverting the relationship given by Eq. (21) yields

$$
k(r)=|\mathbf{b}(r)|=\frac{1}{2 \pi r} \frac{d}{d r}|\mathbf{B}(r)|=\frac{1}{2 \pi r} \frac{d}{d r} 2 \pi r=\frac{1}{r}
$$

Alternatively, using Eq. (38), we can assume that the disclination charge enclosed is equal to $2 \pi$ and this gives the same result. Thus a comparison can be made between this analytical result and the actual measured lattice curvature (just like we did for $N=5000$ in the hard wall case), Fig. (20). There is perfect agreement between the two curves which leads to the conclusion that a conformal lattice can be thought of as a type of disclination.

\section{Experimental Realizations}

The system with the hard wall confinement could potentially be realized in a number of different physical 
contexts. One possible experimental situation involves a collection of polystyrene beads on a disk, located on the edge of which is an insulated boundary. The beads are then exposed to ionizing radiation and the liberated electrons are sucked out of the system by an electrode, leaving behind a collection of positively charged beads. The cluster can then be annealed to the ground state by shaking the disk.

Alternatively the system could be realized using colloidal particles. For these systems one would have to ensure that the Yukawa screening length was made larger than the system size. By using less polar organic solvents, screening lengths as long as $12 \mu \mathrm{m}$ can be achieved [22]. Thus to realize large systems one would need colloidal particles with diameters of, say, $0.1 \mu \mathrm{m}$.

The system with parabolic confinement is of considerable interest to the field of quantum dots. For review articles discussing the fabrication of quantum dots and the harmonic confining approximation, see [23] and 24] respectively and the references contained.

\section{Other Systems}

There are a number of systems in which lattice curvature is quite prominent; one such system is the growth of crystals in amorphous films 25]. Up until now the suggestion has been that these systems possess a confor- mal geometry because the lattice lines are curved. This work demonstrates that this is not necessarily so. The condition that a system has a conformal geometry is very strict, i.e. the lattice density has to obey Eq. (44). It is possible that these systems contain an excess of disclination charge in the interior, which in turn results in the bending of lattice lines. It would be interesting to reexamine such systems in light of the results of this paper. Even the original experiment which sparked the interest in conformal crystals, the so-called gravity's rainbow structure, has been shown not to be stable [26], meaning that it is likely that the system does not form a perfect conformal crystal. Numerical simulations suggest that the system is composed of domains which are separated by defects [26]; these defects may be the actual cause of the lattice curvature in this system. Other interesting systems in which almost perfect conformal crystals have been generated include ferrofluid foams in magnetic fields [27] and soap foams [28]; in some cases the resulting structure contains internal defects. It is hoped that the present work may give some insight into their role.

\section{Acknowledgments}

One of us (AM) would like to thank EPSRC for financial support. We would also like to thank Matthew Hastings and Paul McClarty for useful discussions.
[1] M.W. Cole, Rev. Mod. Phys. 46, 451 (1974).

[2] A.A. Koulakov and B.I. Shklovskii, Phil. Mag. B 77, 1235 (1998).

[3] A.A. Koulakov and B.I. Shklovskii, Phys. Rev. B 57, 2352 (1998).

[4] V.M. Bedanov and F.M. Peeters, Phys. Rev. B 49, 2667 (1993).

[5] M. Kong, B. Partoens, A. Matulis, and F.M. Peeters, Phys. Rev. E 69, 036412 (2004).

[6] Y.J. Lai and I. L, Phys. Rev. E. 60, 4743 (1999).

[7] W.H. Press, B.P. Flannery, and S.A. Teukolsky, Numerical recipies in $C$, 2nd edition (Cambridge University Press, 1993).

[8] A.D. Polyanin and A.V. Manzhirov, Handbook of Integral Equations (CRC Press, 1998).

[9] P.M. Chaikin and T.C. Lubensky, Principles of Condensed Matter Physics (Cambridge University Press, 1995).

[10] J. Weertman and J.R. Weertman, Elementary dislocation theory (Oxford university press, 1992).

[11] J. Friedel, Physics of defects (R. Balian, M. Kleman and J.P. Poirier (Eds.), Les Houches, 1980).

[12] E. Kroner, Physics of defects, R. Balian, M. Kleman and J.P. Poirier (Eds.) (Les Houches, 1980).

[13] L. Bonsall and A.A. Maradudin, Phys. Rev. B 15, 1959 (1977).

[14] http://www.qhull.org/.
[15] http://www.geomview.org/.

[16] J.F. Nye, Acta Metall. 1, 1953 (1952).

[17] M. Bowick and A. Travesset, J. Phys. A 34, 1535 (2001).

[18] K. Kondo, RAAG Memoirs of the Unifying Study of the Basic Problems in Engineering Science by Means of Geometry, Volume 1 (Gakujutsu Bunken Fukyu-Kay, 1955).

[19] F. Rothen, P. Pieranski, N. Rivier, and A. Joyet, Euro. J. Phys. 14, 227 (1993).

[20] F. Rothen and P. Pieranski, Phys. Rev. E 53, 2828 (1996).

[21] H. Jeffreys and B.S. Jeffreys, Methods of Mathematical Physics (Cambridge at the University Press, 1950).

[22] A. Yethiraj and A. van Blaaderen, Nature 421, 513 (2003).

[23] Y. Alhassid, Reviews of Modern Physics 72, 895 (2000).

[24] S.M. Reimann and M. Manninen, Reviews of Modern Physics 74, 1283 (2002).

[25] V.Y. Kolosov and A.R. Tholen, Acta mater. 48, 1829 (2000).

[26] K. Wojciechowski and J. Klos, J. Phys. A 29, 3963 (1996).

[27] F. Elias, J.C. Bacri, F.H. de Mougins, and T. Spengler, Phil. Mag. Lett. 79, 389 (1999).

[28] W. Drenckhan, D. Weaire, and S.J. Cox, Eur. J. Phys. 25, 429 (2004). 